\title{
A study of frequency band structure in two-dimensional homogeneous anisotropic phononic $\mathrm{K}_{3}$-metamaterials
}

\author{
V. N. Gorshkova,, N. Navadeh ${ }^{\mathrm{c}}$, A. S. Fallah ${ }^{\mathrm{c} *}$ \\ a Building 7, Department of Physics, National Technical University of Ukraine- Kiev Polytechnic Institute, \\ 37 Peremogy Avenue, Kiev-56, 03056, Ukraine \\ b MS 213, Los Alamos National Laboratory, Los Alamos, New Mexico 87545, USA \\ c ACEX Building, Department of Aeronautics, South Kensington Campus, Imperial College London, London \\ SW7 2AZ, UK
}

\begin{abstract}
Phononic metamaterials are synthesised materials in which locally resonant units are arranged in a particular geometry of a substratum lattice and connected in a predefined topology. This study investigates dispersion surfaces in two-dimensional anisotropic acoustic metamaterials involving mass-in-mass units connected by massless springs in $\mathrm{K}_{3}$ topology. The reasons behind the particular choice of this topology are explained. Two sets of solutions for the eigenvalue problem $\left|\boldsymbol{D}\left(\omega^{2}, \boldsymbol{k}\right)\right|=$ 0 are obtained and the existence of absolutely different mechanisms of gap formation between acoustic and optical surface frequencies is shown as a bright display of quantum effects like strong coupling, energy splitting, and level crossings in classical mechanical systems. It has been concluded that a single dimensionless parameter i.e. relative mass controls the order of formation of gaps between different frequency surfaces. If the internal mass of the locally resonant mass-in-mass unit, $m$, increases relative to its external mass, $M$, then the coupling between the internal and external vibrations in the whole system rises sharply, and a threshold $\mu^{*}$ is reached so that for $m / M>\mu^{*}$ the optical vibrations break the continuous spectrum of "acoustic phonons" creating the gap between them for any value of other system parameters. The methods to control gap parameters and polarisation properties of the optical vibrations created over these gaps were investigated. Dependencies of morphology and width of gaps for several anisotropic cases have been expounded and the physical meaning of singularity at the point of tangential contact between two adjacent frequency surfaces has been provided. Repulsion between different frequency band curves, as planar projections of surfaces, has been explained. The limiting case of isotropy has been discussed and it has been shown that, in the isotropic case, the lower gap always forms, irrespective of the value of relative mass.
\end{abstract}

Keywords: Phononic metamaterial, dispersion surface, Bloch's theorem, $\mathrm{K}_{3}$ topology, acoustic mode, optical mode, Brillouin zone

\footnotetext{
* To whom correspondence should be addressed:

Tel.: +44 (0) 2075945140

Email: as3@imperial.ac.uk (Arash S. Fallah)
} 


\section{Introduction}

Synthesised materials possessing architected microstructures are crucial in elastic band gap formation. A phononic metamaterial is such a material possessing an artificial microstructure. As such, the metamaterial exhibits unusual response characteristics not readily observed in natural materials and offers certain, potentially beneficial, features of behaviour when vibration mitigation, wave manipulation or sound attenuation are of concern. Unlike natural materials, the constitutive mechanical behaviour of a phononic metamaterial is not determined by its atomic structure but rather by its unit or primitive cell.

A number of researchers have been engaged in investigating electromagnetic properties of photonic metamaterials [1-8] exhibiting unusual properties such as a negative refractive index in order to exploit these for various novel applications [9-11]. However; phononic metamaterials have recently started to attract attention in the fields of acoustics and applied mechanics [12-16]. One of the properties of a phononic metamaterial, particularly of interest in acoustic applications, is the possibility to achieve, simultaneously, negative mass density and elastic modulus $[14,17,18]$ in the strict sense of the effective medium theory $[8,18,19]$. This is analogous to the negative refractive index observed in their photonic counterparts $[4,7,8,20]$. The existence of a phononic band gap, i.e. an interval of frequencies over which mechanical waves cannot propagate, is a direct consequence of this property and is of interest to engineers designing phononic devices ${ }^{\dagger}$. Practical applications of such phononic devices include mechanical filters, vibration isolators, and acoustic waveguides and have been addressed by researchers $[17,19]$.

The studies conducted on phononic band structure encompass those conducted in real space as well as in reciprocal space. To mention but a few, Kushwaha et al. [14, 15] provided one of the earliest calculations of acoustic band gaps in a simple periodic composite. Nevertheless, their calculations were limited to the case of anti-plane shear. Zalipaev et al. [21] also considered anti-plane shear and studied the transition from two-dimensional (2D) wave propagation through the square periodic structure in time-harmonic case to a discrete parameter model of a 2D lattice with masses connected by springs. Martinsson [22] provided a simple method to calculate band gaps with special attention paid to the connection between microstructural geometry and the presence of band gaps. Furthermore, using a phononic lattice structure, complete acoustic band gaps were demonstrated by Martinsson and Movchan [16]. Lumped-mass method for the study of band structure in 2D phononic crystals was considered by Wang et al. [23]. They presented a lumped-mass model, based on the discretization of a continuous system, which worked in the direct space (r-space) and allowed computing the band

\footnotetext{
+ The phenomenon of filtering in phononic devices could also be due to Bragg diffraction. The study of such cases falls beyond the scope of the present study.
} 
structures of 2D phononic crystals. Li and Chan [24] studied doubly negative acoustic metamaterials in which concurrent negative effective density and bulk modulus were obtained. Their doublenegative acoustic system is an acoustic analogue of Veselago's medium in electromagnetism [6, 7, 25], and shares with it many principle features, as negative refractive index, as a consequence of its microstructural composition. Huang and Sun [19] studied wave attenuation mechanisms in acoustic metamaterials of negative effective mass density. The metamaterial under consideration consisted of locally resonant mass-in-mass units which when homogenized had negative effective density. Any such homogenization theory allows for obtaining coarse-scale variation of field variables associated with a heterogeneous medium when the scale ratio, i.e. the ratio between fine and coarse scales, tends to zero while essential features are restored and represented faithfully. Locally resonant sonic materials were also studied by Liu et al. [26]. They fabricated sonic crystals, based on the idea of localized resonant structures, which exhibited spectral gaps with a lattice constant two orders of magnitude smaller than the relevant wavelength. This implied Bragg diffraction was not of interest.

Besides the studies conducted on wave propagation in lattices in the direct space (r-space), the reciprocal lattice formulation (formulation in $\mathbf{k}$-space) is employed extensively by researchers [2730]. There are several advantages associated with employing the $\mathbf{k}$-space formulation. Kittel [31], Brillouin [32], Born [33], Sutton [34] and many other standard textbooks on solid state physics contain the details of the problem formulation in k-space. In a rather recent study, Phani et al. [27] investigated plane wave propagation in infinite 2D periodic lattices using Bloch's theorem. They formulated the exact finite element model of the problem using Timoshenko beam elements possessing distributed masses thus extracted frequency band gaps and examined spatial filtering phenomena in four representative planar lattice topologies viz. hexagonal honeycomb, Kagomé lattice, triangular, and square honeycombs. The plane-wave expansion method was used and the admissible plane wave solution was assumed attenuation-free which rendered Floquet-Bloch's theorem applicable. This method was used by Yang et al [28] to formulate the frequency filtering phenomenon in heterogeneous lattices and by Sigmund and Jensen [35] to show the dependence of the band gap on topology.

More recently researchers have used 3D printing capabilities to confirm the existence of acoustic band gaps experimentally in locally-resonant metastructures [36]. An interesting feature of such phononic metamaterials is the possibility to tailor the band structure by altering the inertial and stiffness properties of elements at a single node in a realistic interval. Band gaps in $1 \mathrm{D}$ and $2 \mathrm{D}$ single- and multi-resonator metamaterials have been studied and parametric studies have depicted this dependence $[18,30]$. An overview of range of filterable frequencies was given recently [37]. 
The studies conducted are limited to extraction of gaps and do not discuss in detail the morphology of frequency surfaces especially of the physical meaning of points of singularity and undefined polarisation. Furthermore, most works of literature do not draw a parallel between quantum mechanical effects and analogous effects observed in classical systems. The effects of anisotropy and the importance of critical mass ratio at which the optical vibrations break the continuous spectrum of "acoustic phonons" creating the gap between them for any value of other system parameters have not been discussed and remain obscure in the literature.

The objective of the present study is to investigate band structure and morphology of dispersion surfaces in anisotropic homogeneous $2 \mathrm{D}$ acoustic metamaterials comprising locally resonant mass-inmass units connected by springs in the simple topology of a complete graph on three vertices $\left(\mathrm{K}_{3}\right)$. Besides simplicity and adequacy of static redundancy, this topology is selected due to two associated facts which have been presented as well-known mathematical theorems in this work. In section 2 the lattice system under consideration has been mathematically defined using the terminology of graph theory, a lingo suitable for the task. This allows the representation of the anisotropic $2 \mathrm{D}$ acoustic metamaterial as an infinite medium consisting of lumped masses and discrete stiffness elements. The internal springs have a particular orientation thus different directional characteristics (source of anisotropy). Equations of motion for the generic single node in the phononic metamaterial have been derived in section 3. Floquet-Bloch's principle is applied and the eigenvalue problem has been derived to study the frequency surfaces of the 2D lattice. In section 4 three primary cases and one asymptotic case have been considered. As the wave vector is assumed to be attenuation-free, the position of the node is immaterial and the change in the complex wave amplitude across a unit cell is uniform throughout the domain. The results obtained show the existence and the extent of the phenomenon of frequency filtering in this class of structures. In some cases singularities have been encountered which have been physically expounded using the morphology of surfaces. Furthermore, for the sake of the present study non-dimensional parameters are extracted and utilised and the functional dependence of band structure on dimensionless parameters observed is discussed. Thresholds on certain parameters are also set which have a particular physical meaning. The study is concluded in section 5 .

\section{The discrete parameter 2D metamaterial}

The building block of the metamaterials considered is the complete graph on $n=3$ vertices $\left(K_{3}\right)$ the schematic of which is shown in Fig.1(a). The schematic of the discrete parameter 2D infinite lattice made of $\mathrm{K}_{3}$ units and consisting of locally resonant nodes is depicted in Fig.1 (b) where the blue vertices consist of internal and external masses (see Fig 2(a)). Fig.1(c) shows the unit cell along with lattice vectors. As denoted in Fig. 1(b) the degree of each vertex in the infinite lattice is 6 . 


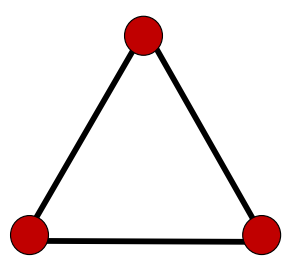

(a)

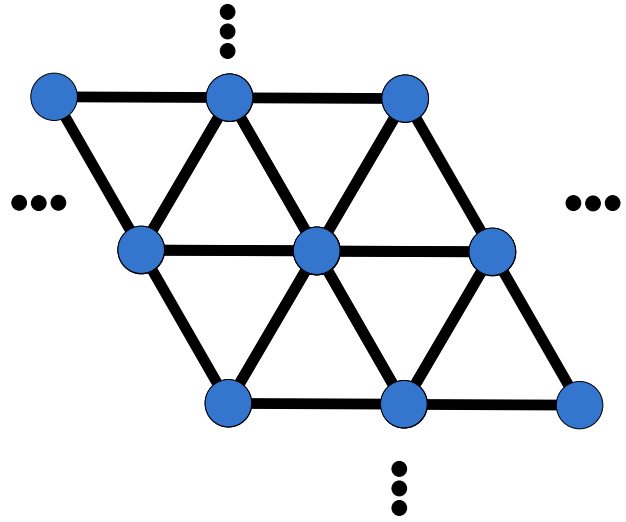

(b)

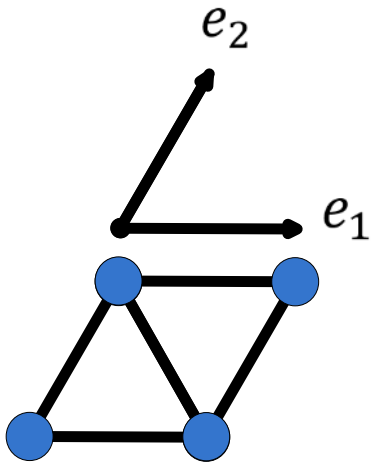

(c)

Fig. 1: (a) the complete graph on three vertices $\left(\mathrm{K}_{3}\right)$ (b) The schematic of the infinite lattice $\mathrm{K}_{3}$, (c) the unit cell along with associated lattice vectors

The entire metamaterial could then be constructed by replicating the nodes along the lattice vectors and connecting them using external springs in the $\mathrm{K}_{3}$ topology. Since, along with topology, the metric properties are of importance lattice constants are defined to refer to the directional distances between primitive/unit cells. The reticulated structure could analogously be obtained through the tessellation of the primitive cell along the finite number of fixed predefined directions (lattice vectors) at particular distances (lattice constants). The $\mathrm{K}_{3}$ lattice metamaterials could, as such, be thought of as essentially a triangular honeycomb with internal nodal resonators. The reasons for the choice of this particular type of topology are as follows:

(1) A triangulated medium is simple to construct and possesses enough degrees of static indeterminacy to be deemed a suitable medium for load transfer (in the case of a truss with hinged connections $\gamma_{s}(G)=N_{i}(G)-M_{c}(G)$ where $\gamma_{s}(G)$ is the degree of static indeterminacy, $N_{i}(G)$ is the number of internal nodes including crossings, and $M_{c}(G)$ is the number of members required to triangulate the entire system provided the connections are pinned [38]. For rigid connections the equation for a plane frame i.e. $\gamma_{s}(G)=3 b_{1}(G)=$ $3\left(M(G)-N(G)+b_{0}(G)\right)$ must be used where $M(G)$ is the number of elements, $N(G)$ the number of nodes and $b_{0}(G)$ and $b_{1}(G)$ designate Betti's zeroth and first numbers, respectively [38]) .

(2) Theorem 1: Every maximal planar graph is fully triangulated (see [39] for proof). This implies triangulated graphs are of particular significance and could be a suitable point of departure for the study of wave propagation in a discrete parameter medium.

(3) Theorem 2: Every simple planar graph is rectilinear (see [40] for proof). This expresses the fact that every planar graph possessing no loops or multiple edges can be drawn in plane using straight lines. 
Together the two theorems imply that the fully-triangulated graph is maximal planar and rectilinear. This means addition of any further edges requires crossings and overlapping edges which implies the implementation of a more intricate procedure of construction. Furthermore, the graph constructed does not need any curved edges to be used.

The structure of the phononic metamaterials could be shown as in Fig. 2. The internal structure of a single phononic node and its connectivity to the external mass is shown in Fig 2(a). Fig. 2(b) shows the connectivity of the external mass to the rest of the medium. Fig. 3 shows the degrees of freedom for the internal mass as well as the centre of mass of the external mass.

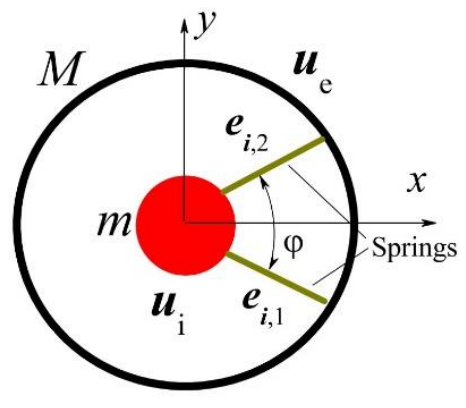

(a)

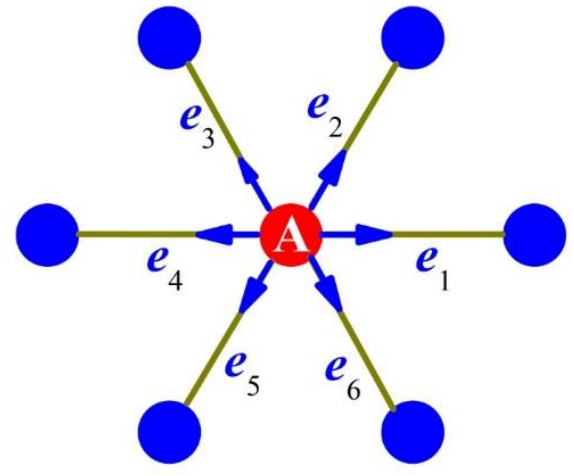

(b)

Fig. 2: The internal and external structures of a generic node indexed (A). (a) the internal structure, (b) the red circle depicts the reference cell shown in (a). Six neighbouring nodes exert forces on the reference cell (node) in (b).

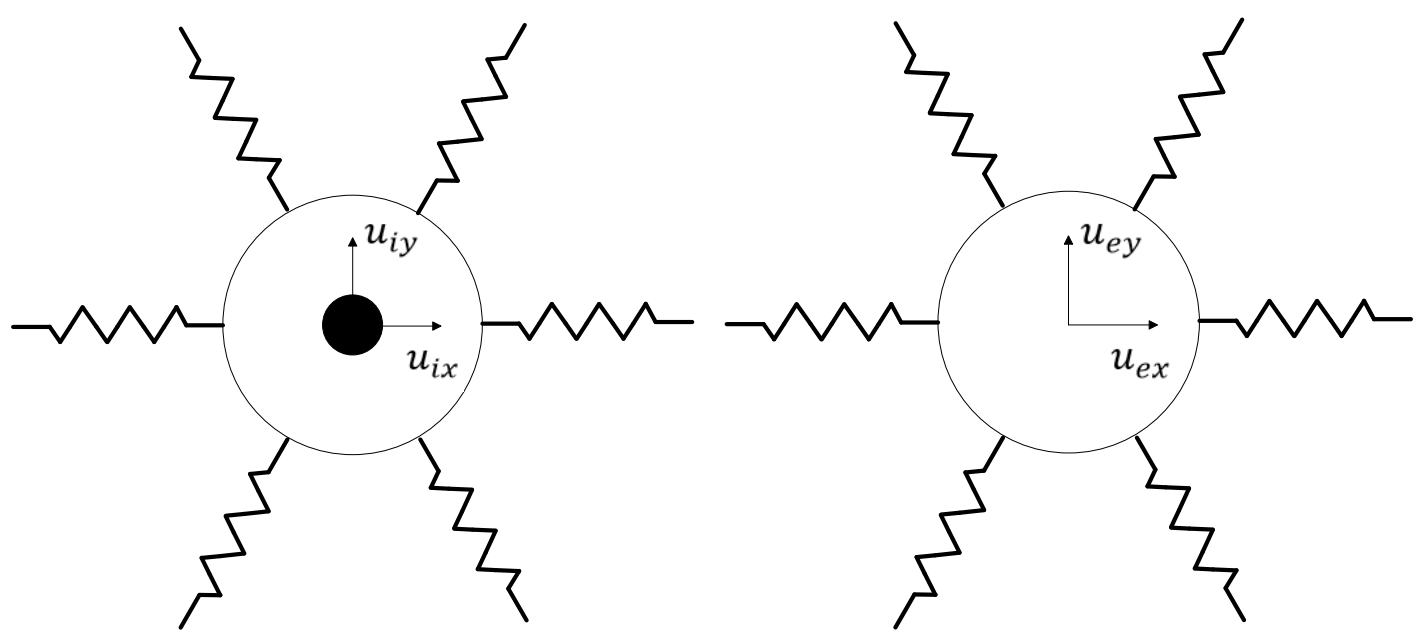

(a)

(b)

Fig. 3: Degrees of freedom for (a) internal mass, (b) centre of mass of the external mass 
The microstructural arrangement of a node could be related to a real situation when a hard mass is buried in a soft massless matrix which, in its own right, is placed inside a hard shell (see Fig. 4). If the external shell is spherical (circular in 2D) isotropy in ensued. However, if a non-spherical (e.g. ellipsoidal (elliptical in 2D)) external shell is assumed anisotropic behaviour emerges (see Fig. 5). In the discrete parameter model of this work anisotropy is considered through allowing the angle between internal springs to be unequal to $\pi / 2$. External anisotropy is achievable through different directional lattice constants. This has not been considered in the present work.

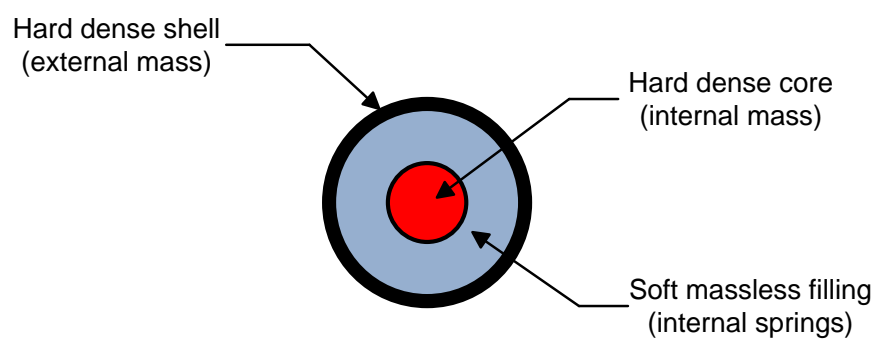

Fig. 4: A representative isotropic locally resonant cell
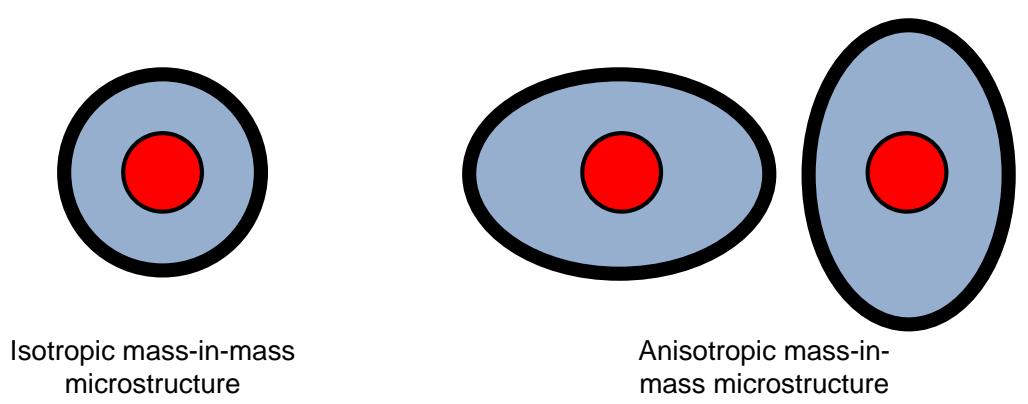

Fig. 5: Representative isotropic and anisotropic locally resonant cells

Spring stiffness for the internal springs could be obtained through analytical, numerical or experimental means. Fig. 6 shows evolution of stresses in a local resonator or locally resonant phononic node (internal spring's elastic response) when the internal mass displaces in horizontal and vertical directions. On calculating the surface integrals of these tractions on the inner core surface one obtains the force the components on which could be related to the corresponding displacement components through directional stiffnesses.

Once the model parameters are obtained the study of band structure in metamaterials could commence. The following section deals with the derivation of equations of motion for the $\mathrm{K}_{3}$ metamaterial and associated analyses for the derivation of dispersion surfaces. 


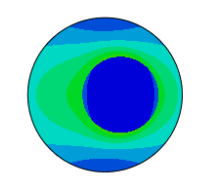

von Mises stress contour

(a)

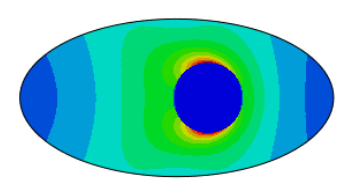

von Mises stress contour

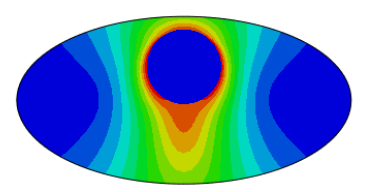

von Mises stress contour

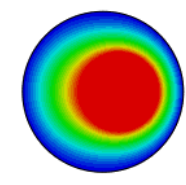

displacement contour

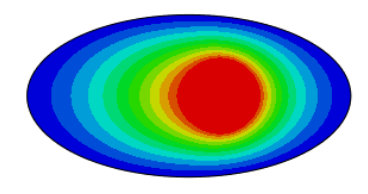

displacement contour

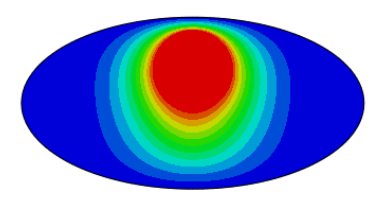

displacement contour

(b)

Fig. 6: Schematic of von Mises stress and displacement fields in the internal soft medium due to directional unit displacement of the internal mass for (a) isotropic case, (b) anisotropic case

\section{Analyses}

\subsection{Derivation of the equations of motion}

Considering the free body diagram of a single phononic node (a mass-in-mass unit) with its connectivity depicted as in Fig. 2(b) the equations of motion could be written as follows:

$$
\begin{gathered}
M \ddot{\boldsymbol{u}}_{e}^{(A)}=\boldsymbol{F}_{\text {ext }}-\boldsymbol{f}_{\text {int }} \\
m \ddot{\boldsymbol{u}}_{i}^{(A)}=\boldsymbol{f}_{\text {int }} \\
\boldsymbol{F}_{\text {ext }}=\sum_{j=1}^{6} \boldsymbol{f}_{j},
\end{gathered}
$$

Where $\boldsymbol{F}_{\text {ext }}$ is the external force exerted on the reference cell, $\boldsymbol{f}_{j}$ the force exerted on the reference cell by a neighbouring cell indexed $j$ and $\boldsymbol{f}_{\text {int }}$ the force applied on the internal mass by the shell.

$$
\boldsymbol{f}_{j}=\chi_{e} \boldsymbol{e}_{j}\left[\boldsymbol{e}_{j} \cdot\left(\boldsymbol{u}_{e}^{(j)}-\boldsymbol{u}_{e}^{(A)}\right)\right]
$$

The unit vector $\boldsymbol{e}_{j}$ is aligned along the spring connecting the reference cell to its $j$-th adjacent cell, $(j=1,2, \ldots, 6)$ and is directed from the ref. cell A to the $j$-th cell (the distance between cells equals $a) \cdot \chi_{e}$ is the stiffness of the external springs.

$$
\boldsymbol{e}_{j}=\left(\cos \alpha_{j}, \sin \alpha_{j}\right), \alpha_{j}=\frac{\pi}{3}(j-1), \quad j=1,2, \ldots, 6
$$


According to the Bloch's theorem for a harmonic plane wave solution:

$$
\boldsymbol{u}_{e}^{(j)}=\boldsymbol{u}_{e}^{(A)} \exp \left(i a \boldsymbol{k} \cdot \boldsymbol{e}_{j}\right)
$$

where $\boldsymbol{k}$ is real wave vector (attenuation-free wave).

Analogously, $\boldsymbol{f}_{i n t}=\chi_{i} \sum_{n=1}^{2} \boldsymbol{e}_{i, n}\left[\boldsymbol{e}_{i, n} \cdot\left(\boldsymbol{u}_{e}^{(A)}-\boldsymbol{u}_{i}^{(A)}\right)\right]$, where $\chi_{i}$ is the stiffness of the internal spring and $\boldsymbol{e}_{i, 1}=\left(\cos \frac{\varphi}{2},-\sin \frac{\varphi}{2}\right), \boldsymbol{e}_{i, 2}=\left(\cos \frac{\varphi}{2}, \sin \frac{\varphi}{2}\right)$.

Substituting expressions obtained for $\boldsymbol{f}_{\text {int }}$ and $\boldsymbol{F}_{\text {ext }}$ into Eq. (1) and taking into account the relation (4) the following equations are obtained for a harmonic wave of frequency $\Omega$ (i. e. $\left.\boldsymbol{u}_{e}^{(A)}=\widehat{\boldsymbol{u}}_{e}^{(A)} \exp (i \Omega t)\right)$ :

$$
\begin{gathered}
-\Omega^{2} m \widehat{\boldsymbol{u}}_{i}^{(A)}=\chi_{i} \sum_{n=1}^{2} \boldsymbol{e}_{i, n}\left[\boldsymbol{e}_{i, n} \cdot\left(\widehat{\boldsymbol{u}}_{e}^{(A)}-\widehat{\boldsymbol{u}}_{i}^{(A)}\right)\right], \\
-\Omega^{2} M \widehat{\boldsymbol{u}}_{e}^{(A)}=\chi_{e} \sum_{j=1}^{6} \boldsymbol{e}_{j}\left(\boldsymbol{e}_{j} \cdot \widehat{\boldsymbol{u}}_{e}^{(A)}\right)\left(\exp \left(i a \boldsymbol{k} \cdot \boldsymbol{e}_{j}\right)-1\right)-\chi_{i} \sum_{n=1}^{2} \boldsymbol{e}_{n}\left[\boldsymbol{e}_{n} \cdot\left(\widehat{\boldsymbol{u}}_{e}^{(A)}-\widehat{\boldsymbol{u}}_{i}^{(A)}\right)\right] .
\end{gathered}
$$

One can easily calculate the eigenfrequencies of the isolated node when there are no external interactions $\boldsymbol{F}_{\text {ext }}=\mathbf{0}$.

$$
\begin{aligned}
f_{\text {int }, x} & =\chi_{i}\left(\sum_{n=1}^{2} \boldsymbol{e}_{i, n}\left[\boldsymbol{e}_{i, n} \cdot\left(\boldsymbol{u}_{e}^{(0)}-\boldsymbol{u}_{i}^{(0)}\right)\right]\right)_{x}= \\
& =2 \chi_{i} u_{e x}^{(0)} \cos ^{2} \frac{\varphi}{2}-2 \chi_{i} u_{i x}^{(0)} \cos ^{2} \frac{\varphi}{2}=\frac{3}{2} \chi_{i} u_{e x}^{(0)}-\frac{3}{2} \chi_{i} u_{i x}^{(0)} \\
f_{\text {int }, y} & =\chi_{i}\left(\sum_{n=1}^{2} \boldsymbol{e}_{i, n}\left[\boldsymbol{e}_{i, n} \cdot\left(\boldsymbol{u}_{e}^{(0)}-\boldsymbol{u}_{i}^{(0)}\right)\right]\right)_{y}= \\
& =2 \chi_{i} u_{e y}^{(0)} \sin ^{2} \frac{\varphi}{2}-2 \chi_{i} u_{i y}^{(0)} \sin ^{2} \frac{\varphi}{2}=\frac{3}{2} \chi_{i} u_{e y}^{(0)}-\frac{3}{2} \chi_{i} u_{y}^{(0)}
\end{aligned}
$$

for $\varphi=\pi / 3$. Using the relation $M \boldsymbol{u}_{e}^{(0)}+m \boldsymbol{u}_{i}^{(0)}=0$ for free vibration of a single node, from Eqs. (5) and (7a) the eigenfrequency of vibration along $x$-axis is obtained as follows:

$$
\lambda_{x}^{2}=2 \frac{\chi_{i}}{m} \cos ^{2} \frac{\varphi}{2}\left(1+\frac{m}{M}\right)=\frac{3}{2} \omega_{0}^{2}\left(1+\frac{m}{M}\right)
$$

Where the value $\omega_{0}^{2}=\frac{\chi_{i}}{m}$ is used as a reference frequency with respect to which frequencies could be normalised.

Analogously, the eigenfrequency of free vibration along $y$-axis is obtained as follows:

$$
\lambda_{y}^{2}=2 \frac{\chi_{i}}{m} \sin ^{2} \frac{\varphi}{2}\left(1+\frac{m}{M}\right)=\frac{1}{2} \omega_{0}^{2}\left(1+\frac{m}{M}\right) .
$$

In both cases $\lambda^{2 \prime} s$ depict the directional resonance frequencies.

In fact, the set of equations (5) and (6) gives the dispersion equation in terms of $\hat{u}_{e x}^{(A)}, \hat{u}_{e y}^{(A)}, \hat{u}_{i x}^{(A)}, \hat{u}_{i y}^{(A)}$, for the reference cell (A), which can be re-written as follows: 


$$
\boldsymbol{D}\left(\Omega^{2}, \boldsymbol{k}\right) \boldsymbol{u}=\mathbf{0}, \quad \boldsymbol{u}=\left[\begin{array}{l}
\boldsymbol{u}_{e} \\
\boldsymbol{u}_{i}
\end{array}\right] \equiv\left[\begin{array}{l}
\widehat{\boldsymbol{u}}_{e}^{(A)} \\
\widehat{\boldsymbol{u}}_{i}^{(A)}
\end{array}\right], \quad\left|\boldsymbol{D}\left(\Omega^{2}, \boldsymbol{k}\right)\right|=\mathbf{0}, \quad \Omega^{2}=\Omega^{2}(\boldsymbol{k}),
$$

where $\boldsymbol{D}\left(\Omega^{2}, \boldsymbol{k}\right)$ is the dynamic matrix which is, in this case, a square $4 \times 4$ matrix. Below, we introduce the non-dimensional frequency as:

$$
\omega^{2}=\Omega^{2} / \omega_{0}^{2}, \quad \omega_{0}^{2}=\frac{\chi_{i}}{m}
$$

and determine the four dispersion surfaces $\omega_{n}^{2}=\omega_{n}^{2}(\boldsymbol{k}), n=1,2,3,4$, which satisfy the equation $\left|\boldsymbol{D}\left(\omega_{n}^{2}(\boldsymbol{k}), \boldsymbol{k}\right)\right|=0$.

Dimensionless parameters of the problem could be constructed based on any pair of the three parameters defined as follows:

$$
\mu=\frac{m}{M}, \quad \beta=\frac{\chi_{i}}{\chi_{e}}, \quad \Omega_{0}^{2}=\mu / \beta=\frac{\chi_{e}}{M} / \omega_{0}^{2} .
$$

Based on Eqs. (5),(6),(7a),(7b) the elements of the dynamic $D_{i j}\left(\omega^{2}, \boldsymbol{k}\right)$ could easily be rewritten using the parameters, $\mu, \Omega_{0}^{2}$, and the dimensionless wave vector $\widehat{\boldsymbol{k}}=\boldsymbol{k} a$ as follows:

$$
\begin{aligned}
& D_{11}=\Omega_{0}^{2}\left[3-2 \cos \left(\hat{k}_{x}\right)-\cos \left(\frac{\hat{k}_{x}}{2}\right) \cos \left(\hat{k}_{y} \frac{\sqrt{3}}{2}\right)\right]+2 \mu \cos ^{2} \frac{\varphi}{2}-\omega^{2}, \\
& D_{22}=3 \Omega_{0}^{2}\left[1-\cos \left(\frac{\hat{k}_{x}}{2}\right) \cos \left(\hat{k}_{y} \frac{\sqrt{3}}{2}\right)\right]+2 \mu \sin ^{2} \frac{\varphi}{2}-\omega^{2}, \\
& D_{33}=2 \cos ^{2} \frac{\varphi}{2}-\omega^{2}, D_{44}=2 \sin ^{2} \frac{\varphi}{2}-\omega^{2}, \\
& D_{12}=D_{21}=\sqrt{3} \Omega_{0}^{2} \sin \left(\frac{\hat{k}_{x}}{2}\right) \sin \left(\hat{k}_{y} \frac{\sqrt{3}}{2}\right), \\
& D_{13}=-2 \mu \cos ^{2} \frac{\varphi}{2}, D_{24}=-2 \mu \sin ^{2} \frac{\varphi}{2}, D_{31}=-2 \cos ^{2} \frac{\varphi}{2}, D_{42}=-2 \sin ^{2} \frac{\varphi}{2}, \\
& D_{14}=D_{23}=D_{32}=D_{34}=D_{41}=D_{43}=0 .
\end{aligned}
$$

\subsection{Extraction of frequency band structure}

As a point of departure in the analysis of dispersion properties a simple reduction is made to the system so that a point-mass lattice is obtained. Such an abstraction provides a benchmark for the subsequent study and renders $\omega_{f}=\omega_{f}(\boldsymbol{k})$ the only dependency of frequency in the system for free vibrations of the lattice when internal masses and springs are excluded. In this case one needs to solve Eq. (9) taking into account expressions (11) at $\mu=0$ and operate only with matrix $\left\|\begin{array}{cc}D_{11} & D_{12} \\ D_{21} & D_{22}\end{array}\right\|$. For this type of vibrations the eigenfunctions are denoted as $\boldsymbol{u}_{f, e}^{(0)}(\boldsymbol{k})$.

It therefore follows that:

$$
\begin{gathered}
\omega_{f, l o w e r}^{2} / \Omega_{0}^{2}=\omega_{f a}^{2}-\omega_{f b}^{2}, \quad \omega_{f, \text { upper }}^{2} / \Omega_{0}^{2}=\omega_{f a}^{2}+\omega_{f b}^{2}, \\
\omega_{f a}^{2}=3-\cos \hat{k}_{x}-2 \cos \frac{\hat{k}_{x}}{2} \cos \frac{\sqrt{3}}{2} \hat{k}_{y},
\end{gathered}
$$




$$
\omega_{f b}^{2}=\sqrt{\left(\cos \hat{k}_{x}-\cos \frac{\hat{k}_{x}}{2} \cos \frac{\sqrt{3}}{2} \hat{k}_{y}\right)^{2}+3 \sin ^{2} \frac{\hat{k}_{x}}{2} \sin ^{2} \frac{\sqrt{3}}{2} \hat{k}_{y}} .
$$

Before proceeding with a discussion of main results obtained, let us demonstrate the structure of free vibrations in the lattice for different branches $\left(\omega_{f, \text { lower }}^{2}(\boldsymbol{k}), \omega_{f, \text { upper }}^{2}(\boldsymbol{k})\right)$ depending on the wave vector $\boldsymbol{k}$.

The main equation of the eigenproblem, i.e. $\boldsymbol{D}(\Omega, \boldsymbol{k}) \boldsymbol{u}=\mathbf{0}$, defines the eigenvectors $\boldsymbol{u}_{n}=$ $\left[\begin{array}{l}\boldsymbol{u}_{e} \\ \boldsymbol{u}_{i}\end{array}\right]_{n}$. The angle between $\boldsymbol{k}$ and $\boldsymbol{u}_{e}$ characterizes the type of vibration for a given $\boldsymbol{k}$ vector. If $\Phi(\boldsymbol{k})=\cos \alpha=\frac{a b s\left[\left(\boldsymbol{k} \cdot \boldsymbol{u}_{e}\right)\right]}{|\boldsymbol{k}|\left|\boldsymbol{u}_{e}\right|} \approx 1$, then the vibration is deemed longitudinal, and in the case of $\cos \alpha \approx$ 0 the vibration is transverse. Fig. 7(a) depicts these two types of free vibration designated by surfaces $\omega_{f, \text { lower }}^{2}(\boldsymbol{k})$ (cyan surface) for transverse vibration and $\omega_{f, \text { upper }}^{2}(\boldsymbol{k})$ (yellow surface) for longitudinal vibration for the normalised dimensionless parameter $\Omega_{0}^{2}=\frac{\mu}{\beta}=\frac{\chi_{e}}{M} / \omega_{0}^{2}=1$. The functional dependence of $\omega^{2}(\boldsymbol{k})$ is represented at the left half of the square (dashed line) shown in Fig. 7(b) - The first Brillouin zone for the hexagonal 2D-lattice. Fig. 7(c) shows the structure of the contours for $\omega_{f, l o w e r}^{2}(\boldsymbol{k})$ at a wider area, which is larger than the first Brillouin zone (cyan coloured in the Fig. 7(c)).

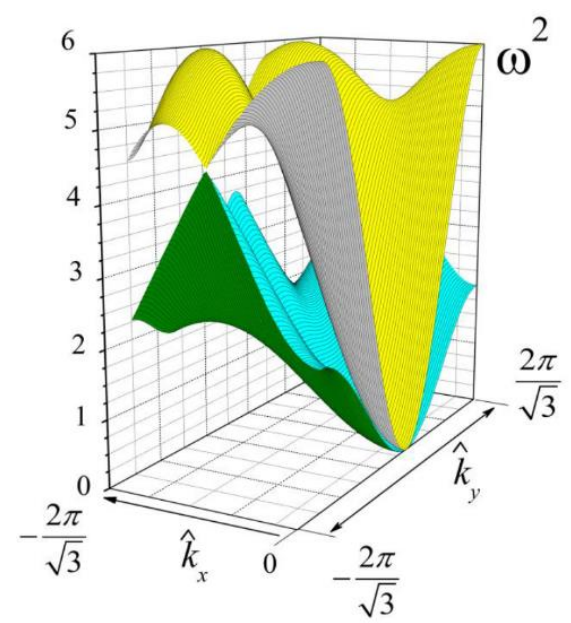

(a)

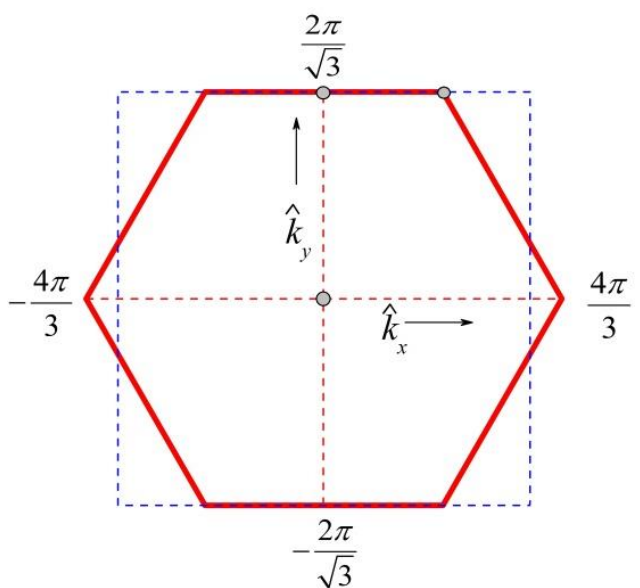

(b)

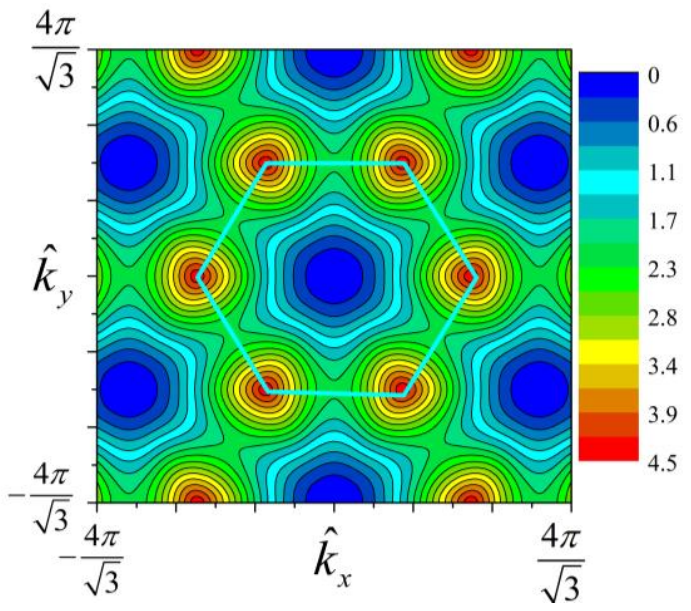

(c) 
Fig. 7: (a) Dispersion surfaces, (b) the first Brillouin zone, (c) 2D mapping of $\omega_{f, \text { lower }}^{2}(\boldsymbol{k})$ on a Brillouin zone and its neighbourhood

Fig. 8 shows both vibration modes as characterized by the essentially isotropic function $\Phi(\boldsymbol{k})=\cos \alpha$ ( $\alpha$ being the angle of polarisation) at the center of the Brillouin zone (according to the general properties of the elastic medium, transverse vibrations lie on the lower frequency-surface and longitudinal vibrations on the upper one). The reason is that the system under consideration has six planes of symmetry, and correspondingly twelve directions of the wave vector $\boldsymbol{k}$ such that exactly one of the conditions $\boldsymbol{u}_{e} \perp \boldsymbol{k}$ or $\boldsymbol{u}_{e} \| \boldsymbol{k}$ holds. These properties need to be known and considered carefully when looking for the parameters to be altered for gap formation between neighbouring frequencysurfaces, which ranges over the first Brillouin zone.

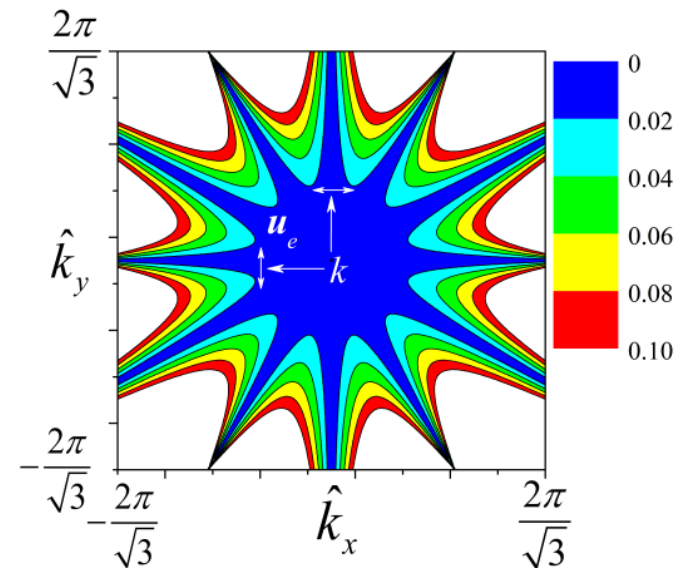

(a)

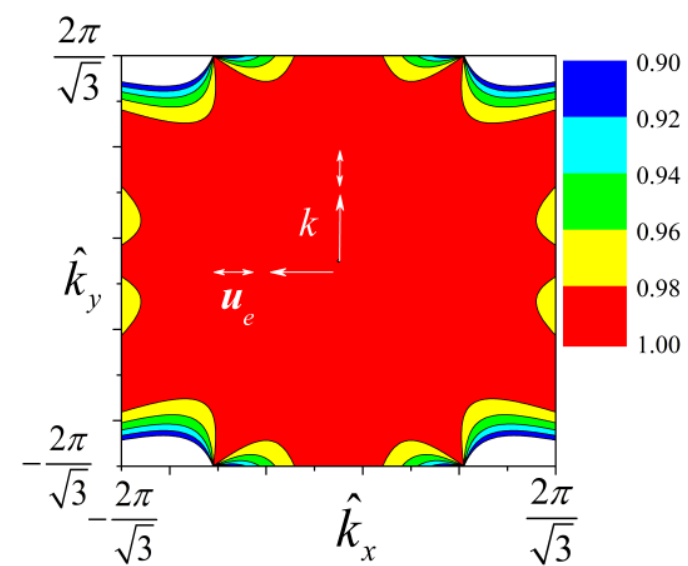

(b)

Fig. 8: The distribution in Brillouin zone ( $\boldsymbol{k}$-space) of the function $\Phi(\boldsymbol{k}) . \Phi(\boldsymbol{k})=\cos \alpha$, where $\alpha$ is the angle between the wave vector $\boldsymbol{k}$ and displacement vector $\boldsymbol{u}_{f, e}^{(0)}(\boldsymbol{k})$ for (a) the lower frequencysurface, $\omega_{f, \text { lower }}^{2}(\boldsymbol{k})$, and (b) the higher frequency-surface, $\omega_{f, \text { upper }}^{2}(\boldsymbol{k})$. The dependency of $\Phi(\boldsymbol{k})$ is presented at the square (dashed line) shown in Fig. 7(b) which is (a) coloured in blue is the region where $\cos \alpha \leq 0.1$; and (b) coloured in red is the region where $\cos \alpha \geq 0.9$.

As in the first approach, we can find the solution to the eigenvalue problem of Eq. (9) for the limiting case when the mass and stiffness ratios vanish while $\omega_{0}^{2}$ remains constant. $(\mu \rightarrow 0$ in Eq. 11 and $\beta \rightarrow$ 0 in Eq. 10 -Fig. 9a). The shown set of the frequency-surfaces in Fig. 9a is, in fact, the combination of the set of four analytically calculated vibration modes of Eqs. (8a),(8b),(12).

$$
\begin{gathered}
\omega_{1}^{2}(\boldsymbol{k})=\omega_{f, \text { lower }}^{2}(\boldsymbol{k}), \omega_{2}^{2}(\boldsymbol{k})=\omega_{f, \text { upper }}^{2}(\boldsymbol{k}), \\
\omega_{3}^{2}(\boldsymbol{k})=\lambda_{y}^{2}=\frac{1}{2}, \omega_{4}^{2}(\boldsymbol{k})=\lambda_{x}^{2}=\frac{3}{2}
\end{gathered}
$$

The calculated vibration modes "don't interact" with each other since the parameter $\mu$ in the matrix $\boldsymbol{D}(\omega, \boldsymbol{k})$ formally describes the correlation between internal and external oscillations. The second pair of planes in (13) cross the first pair of surfaces in 3D i.e. in $\left(k_{x}, k_{y}, \omega^{2}\right)$-space. This can be interpreted as vibration energy-level crossing. For any nonzero value of internal mass $(\mu \neq 0)$, even when $\mu \ll 1$, there arises some interaction between vibrations of different type at the numerous lines of crossing (Fig. 9(a)), and the morphology of surfaces essentially transforms (Fig. 9 (b)) . Just as in quantum mechanics, repulsions of energy levels occur followed by formation of four isolated frequency/energy-surfaces. This repulsion effect is known as "level repulsion" or the Wigner-von Neumann non-crossing (anti-crossing) rule. 


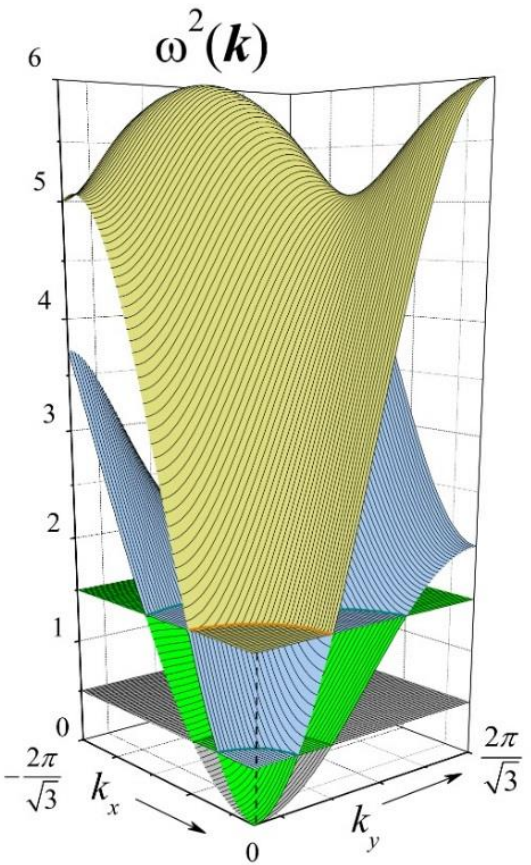

(a)

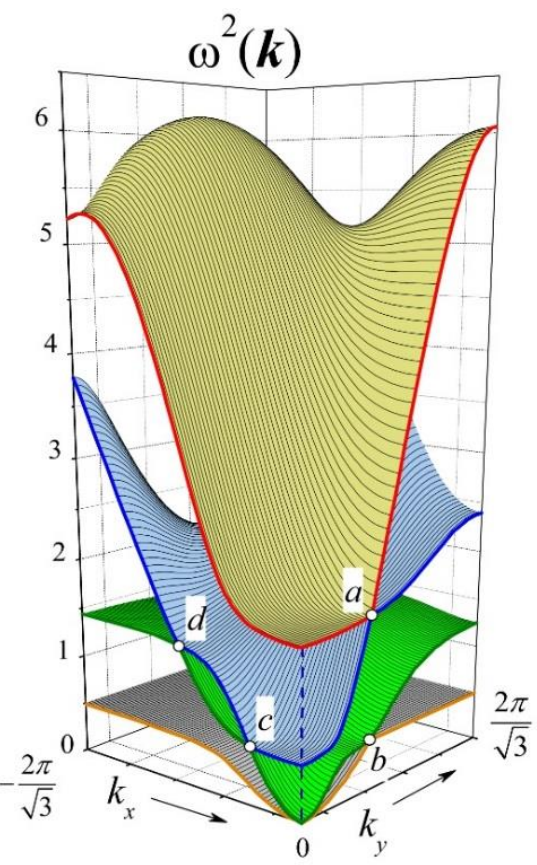

(b)

Fig. 9: (a) Eigensurfaces for the limiting case of $\Omega_{0}^{2}=1, \mu=0$; the frequency-surfaces are calculated and shown for the upper-left quarter of the square region shown in Fig. 7(b). (b) Eigensurfaces at $\Omega_{0}^{2}=1, \mu=0.1$; white circles denote points of contacts between the neighbouring frequency surfaces.

(See [41, 42] for a detailed discussion of the case). For the given set of model parameters corresponding to the latter surface morphology (as in Fig. 9(b)), one can observe that a group of isolated points (here labelled as $a, b, c$, and $d$ ) remain in contact subsequent to occurrence of localised repulsion, i.e. when other areas of adjacent surfaces in the neighbourhood of these points repel each other. At these points the frequency-surfaces contact and the coupling strength between two oscillations of the same frequency at a given $\boldsymbol{k}$ is absent. The physical mechanism responsible for both the repulsion and preservation of morphology encompassing the same points of contact can be qualitatively described using the first approach in the following way.

In the case of the equality of lower and upper vibration frequencies, $\omega_{f, \text { lower,upper }}^{2}=\lambda_{x, y}^{2}$, at a given $\boldsymbol{k}$, the angle of polarization, $\Phi(\boldsymbol{k})$, for both modes of oscillation must be considered. In other words, the angle between two eigenfunctions that describe the displacements of the external mass, $\boldsymbol{u}_{f, e}^{(0)}(\boldsymbol{k}), \boldsymbol{u}_{i n t, e}^{(0)}$ have to be analyzed $\left(\boldsymbol{u}_{f, e}^{(0)}(\boldsymbol{k})\right.$ is the displacement of the external mass as $\mu \rightarrow 0$, $\beta \rightarrow 0$ while $\omega_{0}^{2}=$ const ; and $\boldsymbol{u}_{i n t, e}^{(0)}$ is that of external displacement for $\chi_{e}=0$ ).

There are two extreme cases:

$$
\begin{aligned}
& \boldsymbol{u}_{f, e}^{(0)}(\boldsymbol{k}) \perp \boldsymbol{u}_{i n t, e}^{(0)} \\
& \boldsymbol{u}_{f, e}^{(0)}(\boldsymbol{k}) \| \boldsymbol{u}_{i n t, e}^{(0)}
\end{aligned}
$$

It is obvious that in the first case (Eq. (14a)) the vibration modes don't "interact" and two the frequency-surfaces retain contact (See points $a$ and $b$ in Fig. 10). The free internal vibrations (FIV) possess only two directions of polarization of the displacements, $\boldsymbol{u}_{i n t, e}^{(0)}$ : along $y$-axis with the lower frequency, $\lambda_{y}^{2}$, and along $x$-axis with the higher frequency $\lambda_{x}^{2}$. Contrary to this frequency pair, the free 
external vibrations (FEV) are characterized by rather isotropic functions $\Phi(\boldsymbol{k})$ for both branches of $\boldsymbol{u}_{f, e}^{(0)}(\boldsymbol{k})$ (see Fig. 8). Thus, there are two points on the plane $k_{x}=0$ where Eqs. (15a) and (15b) are valid (See Figs.9(b) and 10).

$$
\begin{array}{ll}
\lambda_{x}^{2}=\omega_{f, \text { upper }}^{2}, & \boldsymbol{u}_{f, e}^{(0)}(\boldsymbol{k}) \perp \boldsymbol{u}_{\text {int }, e}^{(0)} \quad(\operatorname{point} a), \\
\lambda_{y}^{2}=\omega_{f, \text { lower }}^{2}, & \boldsymbol{u}_{f, e}^{(0)}(\boldsymbol{k}) \perp \boldsymbol{u}_{i n t, e}^{(0)}(\text { point } b) .
\end{array}
$$

As a result of (12), the frequencies $\omega_{f, \text { lower }}^{2}, \omega_{f, \text { upper }}^{2}$ at $k_{x}=0$ are equal to

$$
\begin{gathered}
\omega_{f, \text { lower }}^{2}\left(k_{x}=0, k_{y}\right)=\Omega_{0}^{2}\left(1-\cos \frac{\sqrt{3}}{2} \hat{k}_{y}\right), \\
\omega_{f, \text { upper }}^{2}\left(k_{x}=0, k_{y}\right)=3 \Omega_{0}^{2}\left(1-\cos \frac{\sqrt{3}}{2} \hat{k}_{y}\right)
\end{gathered}
$$

and $\omega_{f, \text { upper }}^{2}\left(k_{x}=0\right) / \omega_{f, \text { lower }}^{2}\left(k_{x}=0\right)=\lambda_{x}^{2} / \lambda_{y}^{2}=3$ (See Eqs. (8a) and (8b)).

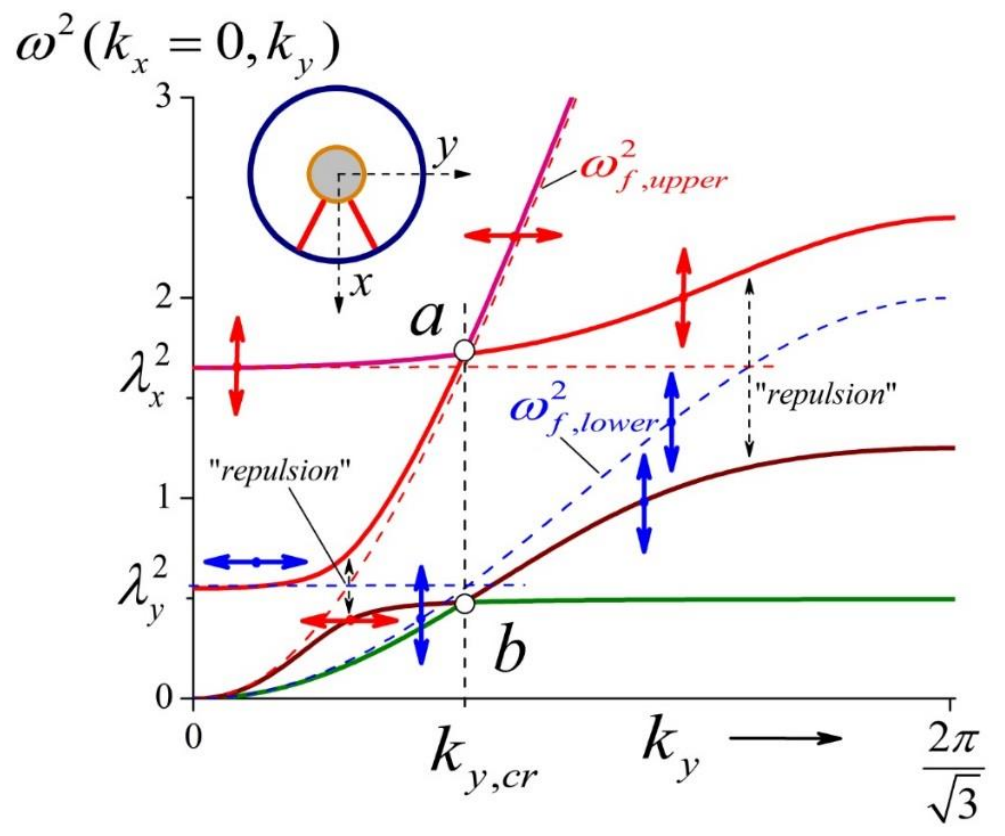

Fig. 10: Cross section of the surfaces shown in Fig. 9(b) by the plane $k_{x}=0$. Arrows denote orientation of the displacements $\boldsymbol{u}_{e}$ relative to the $y$-axis for different types of vibration. The schematic figure inserted at the top reminds the orientation of the internal structure for nodes on the plane $x y$. Red and blue dash lines are cross sections of the four surfaces of Eq. (13). $\lambda_{x}^{2}=$ $1 / 2(1+\mu)=0.55, \lambda_{x}^{2}=3 \lambda_{y}^{2}=1.65$.

Thus both equalities (15a) and (15b) are satisfied for the same value of $k_{y, c r}$ :

$$
k_{y, c r}=\frac{2}{\sqrt{3}} \arccos \left[1-(1+\mu) /\left(2 \Omega_{0}^{2}\right)\right] .
$$

At $\Omega_{0}^{2}=1, k_{y, c r} \approx 1.27$ which corresponds to results shown in Fig. 10. At the regions where $\lambda_{x}^{2}=$ $\omega_{f, \text { lower }}^{2}\left(k_{x}=0, k_{y}\right)$ or $\lambda_{y}^{2}=\omega_{f, \text { upper }}^{2}\left(k_{x}=0, k_{y}\right)$ in both cases $\boldsymbol{u}_{f, e}^{(0)}(\boldsymbol{k}) \| \boldsymbol{u}_{\text {int }, e}^{(0)}$, and one can see the effects of repulsion (See Fig. 10).

In the $\left(k_{x}, \omega\right)$-plane (See Fig. 9b), the equalities $\lambda_{x}^{2}=\omega_{f, \text { upper }}^{2}$ and $\lambda_{y}^{2}=\omega_{f, \text { lower }}^{2}$, are satisfied (in opposite to the $\left(k_{y}, \omega\right)$-plane) at $\boldsymbol{u}_{f, e}^{(0)}(\boldsymbol{k}) \| \boldsymbol{u}_{\text {int, },}^{(0)}$ due to the rotation of the FEV-vectors, 
$\boldsymbol{u}_{f, e}^{(0)}(\boldsymbol{k})-$ See. Fig. 8. - by fixed orientations of the FIV-vectors, $\boldsymbol{u}_{i n t, e}^{(0)}$. Thus, the points of contacts convert to regions of marked repulsions. Such transformations (contact-repulsion) can be easily retraced by comparison with Figs.10 and 11.

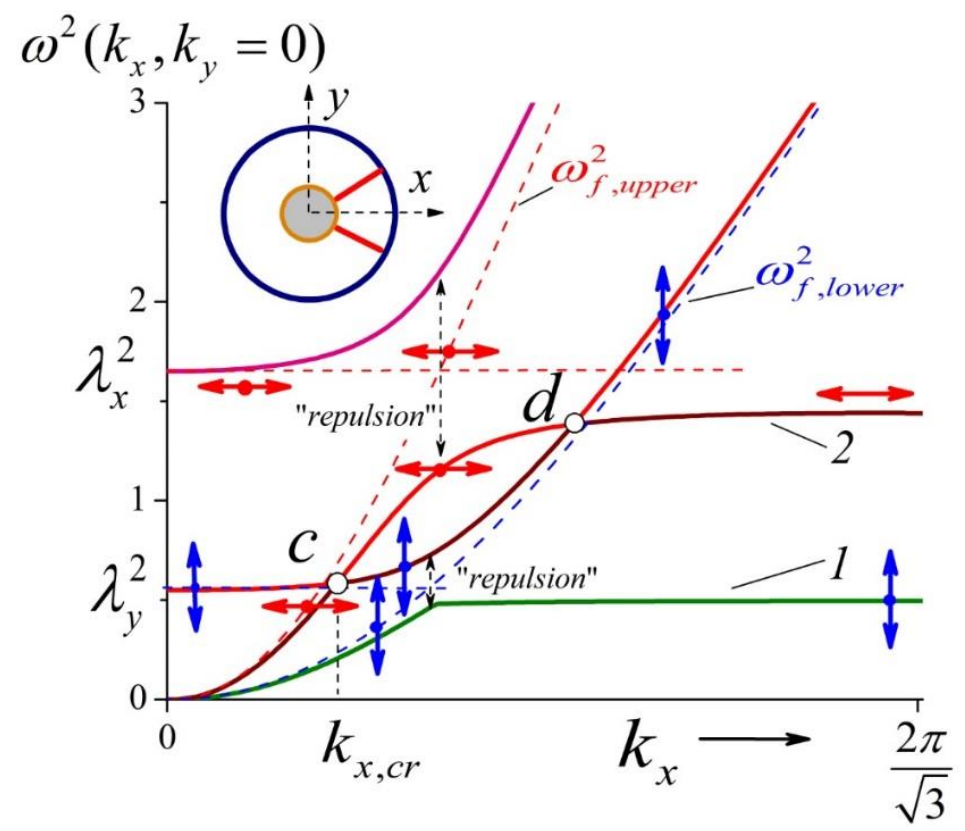

Fig. 11: Cross section of the surfaces shown in Fig. $9 \mathrm{~b}$ by the plane $k_{y}=0$. Arrows denote orientation of the displacements $\boldsymbol{u}_{e}$ relative to the $x$-axis for different types of vibration. The insert remind the orientation of the internal structure of nodes at the plane $x y$.

To elaborate, the repulsion at the region where $\lambda_{x, y}^{2}=\omega_{f, \text { lower,upper }}^{2}$ and $\boldsymbol{u}_{f, e}^{(0)}(\boldsymbol{k}) \| \boldsymbol{u}_{\text {int,e }}^{(0)}$ (where the coupling strength between one of the internal oscillations, $\lambda_{x}^{2}$ or $\lambda_{y}^{2}$ and one of the free external oscillations $\omega_{f, \text { lower }}^{2}(\boldsymbol{k}), \omega_{f, \text { upper }}^{2}(\boldsymbol{k})$ is maximal) can be interpreted as in the sequel.

It is evident that the frequencies of the FIV, $\lambda_{x, y}^{2}$, are proportional to $\left(1+\frac{m}{M}\right)$ (See Eqs. (8a) and (8b)). The multiplier $\left(1+\frac{m}{M}\right)$ reflects the fact that the oscillation of internal and external masses is in antiphase, which increases the effective stiffness of the internal springs, $\chi_{i}^{*}=\chi_{i}\left(1-\frac{\boldsymbol{u}_{i n t, e}^{(0)}}{\boldsymbol{u}_{i n t, i}^{(0)}}\right)>\chi_{i}$, compared to the case of the motionless node shell $\left(\boldsymbol{u}_{e}^{(0)}=0\right.$ in Eq. (7a)). In the first approach, combining the two modes i.e. FIV and FEV at the critical points $\lambda_{x, y}^{2}=\omega_{f, l o w e r, u p p e r}^{2}$ can be realized in two ways:

(i) Anti-phase displacements i.e. $\boldsymbol{u}_{f, e}^{(0)}(\boldsymbol{k}) \uparrow \downarrow \boldsymbol{u}_{i n t, e}^{(0)}$, which implies $\boldsymbol{u}_{e} \approx \boldsymbol{u}_{i n t, e}^{(0)}+$ $\boldsymbol{u}_{f, e}^{(0)}(\boldsymbol{k})<\boldsymbol{u}_{i n t, e}^{(0)}$, and formally corresponds to increasing the effective mass, $M^{*}$, of the node shell, thus $M^{*}>M$;

(ii) In-phase displacements i.e. $\boldsymbol{u}_{f, e}^{(0)}(\boldsymbol{k}) \uparrow \uparrow \boldsymbol{u}_{\text {int,e }}^{(0)}$, which implies $\boldsymbol{u}_{e} \approx \boldsymbol{u}_{\text {int }, e}^{(0)}+$ $\boldsymbol{u}_{f, e}^{(0)}(\boldsymbol{k})>\boldsymbol{u}_{\text {int }, e}^{(0)}$, therefore $M^{*}<M$. 
So, the cases (i) and (ii) are responsible for formation of the lower and the upper frequencies when the repulsion occurs (See. Figs.10 and 11).

It is very useful to determine the type of vibration at the acoustic frequency-surfaces like 1 and 2 in Fig. 11. The results partially presented in Fig. 12 for high parameter $\Omega_{0}^{2}\left(\Omega_{0}^{2}=10\right)$ provide an explanation.

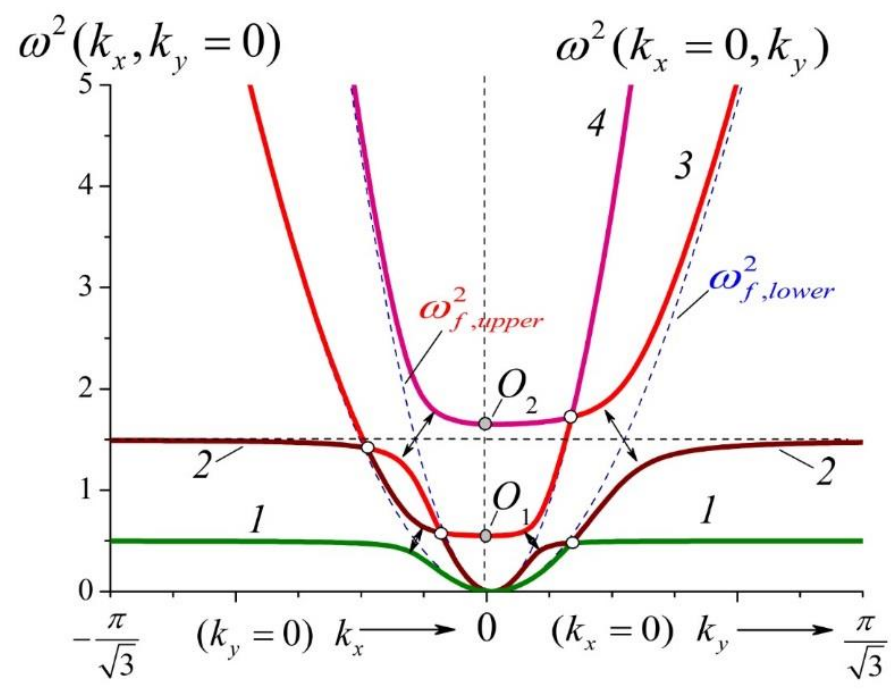

Fig. 12: Cross sections of the frequency-surfaces by the plane $k_{y}=0$ (left half) and by the plane $k_{x}=0$ (right half). $\mu=0.1, \Omega_{0}^{2}=10$ (ten time higher than in Figs.10 and 11). Arrows denote the zones of "levels repulsion". The sections are presented only at the central region of the first Brillion zone.

At the point $\boldsymbol{k}=0$ the lower optical frequency (the point $O_{1}$ ) correspond to the internal oscillations with the frequency $\lambda_{y}^{2}=\frac{1}{2}(1+\mu)=0.55$. These vibrations interact and combine in a sophisticated way with the $\omega_{f, \text { lower }}^{2}(\boldsymbol{k})$-surface (See the left half of Fig. 12) and with the $\omega_{f \text {, upper }}^{2}(\boldsymbol{k})$-surface (See the right half of Fig. 12). In both cases one can see that after repulsion the frequencies of vibrations on surface 1 are practically the same: $\omega_{(1)}^{2}(\boldsymbol{k}) \approx 1 / 2$. A detailed analysis of the corresponding eigen functions indicates that on this surface vibrations occur along $k_{y}$-axis (on the periphery of the first Brillouin-zone) but the displacements of the external masses are at least four hundred times less than displacements of the internal masses (for the free internal vibrations $\left|\frac{\boldsymbol{u}_{i n t, e}^{(0)}}{\boldsymbol{u}_{i n t, i}^{(0)}}\right|=\frac{m}{M}=0.1$ ). Analogously, at point $O_{2}$ on the upper optical surface $\omega^{2}(k=0)=\lambda_{x}^{2}=\frac{3}{2}(1+\mu)=1.65$ but on surface 2 vibrations occur with the frequency $\omega_{(2)}^{2}(\boldsymbol{k}) \approx 3 / 2$ (excluding the central region). Besides, at the periphery of the Brillouin zone the displacements $\boldsymbol{u}_{e}(\boldsymbol{k})$ point to their origin from the free highfrequency optic vibrations. In other words, $\boldsymbol{u}_{e}$ mainly oriented along $x$-axis and the corresponding angle $\gamma=\operatorname{acos}\left(u_{e x} /\left|\boldsymbol{u}_{e}\right|\right)$ does not exceed $32^{\circ}$ (See Fig. 13a). These results can be interpreted in the following way. 


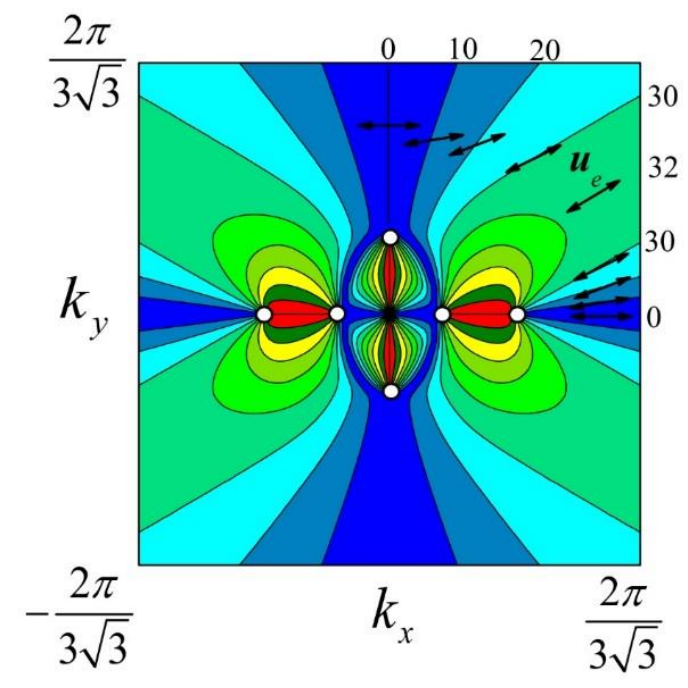

(a)

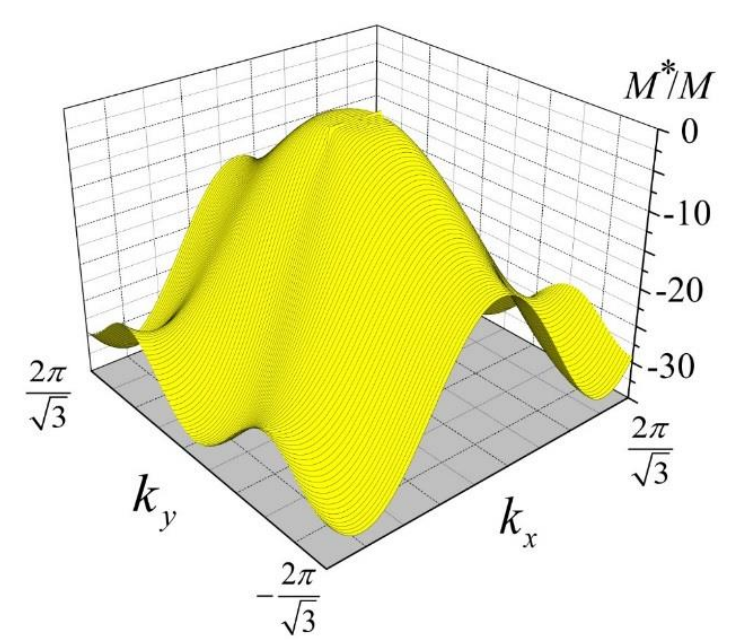

(b)

Fig. 13: Characteristics of the upper acoustic surfaces, $\omega_{(2)}^{2}(\boldsymbol{k})$, at $\mu=0.1, \Omega_{0}^{2}=10$ (See Fig. 12). (a) The distribution of the angle, $\gamma$, between displacements of the node shells, $\boldsymbol{u}_{e}$, and the $x$-axis in the $k_{x}, k_{y}$-space : $\gamma\left(k_{x}, k_{y}\right)=\operatorname{acos}\left(u_{e x} /\left|\boldsymbol{u}_{e}\right|\right)$. The size of the side for the region presented is three time less than the size of the Brillouin zone. The numbers by the equiscalar contour lines indicate the angle $\gamma$ in degrees. White circles correspond to points of tangential contact between the neighbouring frequency surfaces (See Fig. 12). Out of the central area the angle $\gamma$ doesn't exceed the limit of $32^{0}$. (b) The ratio $M^{*} / M$ as a function of $k_{x}, k_{y}$ as $M^{*} / M=-\mu\left|\boldsymbol{u}_{i}\right| /\left|\boldsymbol{u}_{e}\right|, \boldsymbol{u}_{i} \cdot \boldsymbol{u}_{e}>0$.

The external vibratory force field that exerts on the node shells can be brought to an effective mass of the shells in the assumed "free" internal vibrations on the acoustic branches at the periphery of the Brillouin zone. The self-consistency of the external and internal forces results in the two possible effective masses, $M_{1}^{*}, M_{2}^{*}$, for these acoustic surfaces. Similarly to the free internal vibration in which $m / M=\mu=-\boldsymbol{u}_{i n t, e}^{(0)} / \boldsymbol{u}_{i n t, i}^{(0)}\left(\boldsymbol{u}_{i n t, e}^{(0)} \uparrow \downarrow \boldsymbol{u}_{i n t, i}^{(0)}, \boldsymbol{u}_{i n t, e}^{(0)} \cdot \boldsymbol{u}_{\text {int }, i}^{(0)}<\mathbf{0}\right)$, one can estimate the effective masses as follows: $m / M^{*}=-\left|\boldsymbol{u}_{e}\right| /\left|\boldsymbol{u}_{i}\right|$, and $M^{*} / M=-\mu\left|\boldsymbol{u}_{i}\right| /\left|\boldsymbol{u}_{e}\right|$ (in the preceding equations it has been taken into account that in the acoustic wave the internal and the external masses vibrate in-phase, and $\boldsymbol{u}_{i} \cdot \boldsymbol{u}_{e}>0$, which physically corresponds to decreasing the effective stiffness of the internal springs). As shown by the results in Fig. 13b, the effective mass at the periphery of the Brillouin zone is negative and $\left|M^{*}\right| \gg M$ (on the $\omega_{(1)}^{2}(\boldsymbol{k})$-surface the corresponding effective mass goes down to $-120 M$ ). In fact it could be simply argued that the greater the value of $\Omega_{0}^{2}$ (external stiffness) the greater is the absolute value of the effective mass $\left|M^{*}\right|$ due to the fact that increasing external stiffness renders $\boldsymbol{u}_{e}$ small.

The qualitative analysis of the results presented in Figs.12 and 13 leads to the conjecture that the frequencies of the acoustic vibrations never exceed their corresponding thresholds $\lambda_{y}^{2}\left(M^{*}=-\infty\right)$ and $\lambda_{x}^{2}\left(M^{*}=-\infty\right)$, respectively:

$$
\begin{aligned}
& \max \left(\omega_{(1)}^{2}(\boldsymbol{k})\right)=\lambda_{y}^{2}\left(M^{*}=-\infty\right) \\
& \max \left(\omega_{(2)}^{2}(\boldsymbol{k})\right)=\lambda_{x}^{2}\left(M^{*}=-\infty\right)
\end{aligned}
$$


Throughout the numerical analyses conducted the expected behaviour prevailed. For instance, for the case of $\Omega_{0}^{2}=10, \mu=1$ the higher frequency $\omega^{2}(k=0)=\lambda_{x}^{2}=3$ but $\max \left(\omega_{(2)}^{2}(\boldsymbol{k})\right)=$ $1.455<\lambda_{x}^{2}\left(M^{*}=-\infty\right)=1.5$. The ansatz conjectured, i.e. Eq. (19), could be analytically proved as follows.

According to Eq. (8a), $\lambda_{x}^{2}\left(M^{*}=-\infty\right)=2 \cos ^{2} \frac{\varphi}{2}$. The dispersion equation $|\boldsymbol{D}(\omega, \boldsymbol{k})|=0$ at $\omega^{2}=2 \cos ^{2} \frac{\varphi}{2},\left|\boldsymbol{D}\left(\omega^{2}=2 \cos ^{2} \frac{\varphi}{2}, \boldsymbol{k}\right)\right|=0$, takes the following form:

$$
1.5 \Omega_{0}^{2}\left[1-\cos \left(\frac{\hat{k}_{x}}{2}\right) \cos \left(\hat{k}_{y} \frac{\sqrt{3}}{2}\right)\right]=\cos ^{2} \frac{\varphi}{2}\left(\cos \varphi-\mu \sin ^{2} \frac{\varphi}{2}\right) / \cos \varphi .
$$

For an arbitrary direction $\boldsymbol{e}\left(\boldsymbol{e}=e_{x} \boldsymbol{i}+e_{y} \boldsymbol{j}\right.$ being the unit vector) the function

$$
f(k)=\cos \left(\frac{1}{2} \hat{k} e_{x}\right) \cos \left(\frac{\sqrt{3}}{2} \hat{k} e_{y}\right) \equiv \cos \left(\frac{\hat{k}_{x}}{2}\right) \cos \left(\frac{\hat{k}_{y} \sqrt{3}}{2}\right),
$$

is a monotonically decreasing function in the first Brillouin zone. So, the Eq. (20) may have a single solution in terms of $\hat{k}$, and the solution corresponds to a point that can only be situated at the lower "optical" frequency-surface - See. Fig. 12, for example. There exists no solution of Eq. (20) at the acoustic frequency surfaces 2 for any value of the dimensionless wave parameter $\hat{k}$. However, it must be noticed that the lower the ratio $\frac{\mu}{\Omega_{0}^{2}}=\frac{\chi_{i}}{\chi_{e}}=\beta$ the closer is the $\max \left(\omega_{(2)}^{2}(\boldsymbol{k})\right)$ to its threshold $\lambda_{x}^{2}\left(M^{*}=-\infty\right)$.

The statements (19) are very important in finding the gaps needed to be formed by the proper choice of parameters $\mu, \Omega_{0}^{2}$ or equivalently $\mu, \beta$.

\section{Mechanisms of gap formation}

Formation of gaps of different characteristics is of significance in designing phononic devices. Gaps of diverse characteristics, between neighbouring frequency-surfaces, can be formed based on different physical mechanisms, which depend on the value of the dimensionless parameter $\mu$.

For $\mu \ll 1$ a second parameter i.e. $\Omega_{0}^{2}$ must be low enough so that the point of contact of type $a$ (See. Fig. 10) between surfaces vanishes. This means that (i) the upper frequency of the internal frequencies, $\lambda_{x}^{2}=1.5(1+\mu)$ - See. Eq. (8a) - is at least greater than $\max \left\{\omega_{f, \text { upper }}^{2}(\boldsymbol{k})\right\}$, (ii) Eq. (17) doesn't have a solution, that is $\left|1-(1+\mu) /\left(2 \Omega_{0}^{2}\right)\right|>1$. Finally, the upper gap (between the frequency surfaces $3-4$, Fig. 14a) appears if

$$
\Omega_{0}^{2}<\frac{1+\mu}{4}
$$

So, $\Omega_{0}^{2}<0.275$ if $\mu=0.1$. 


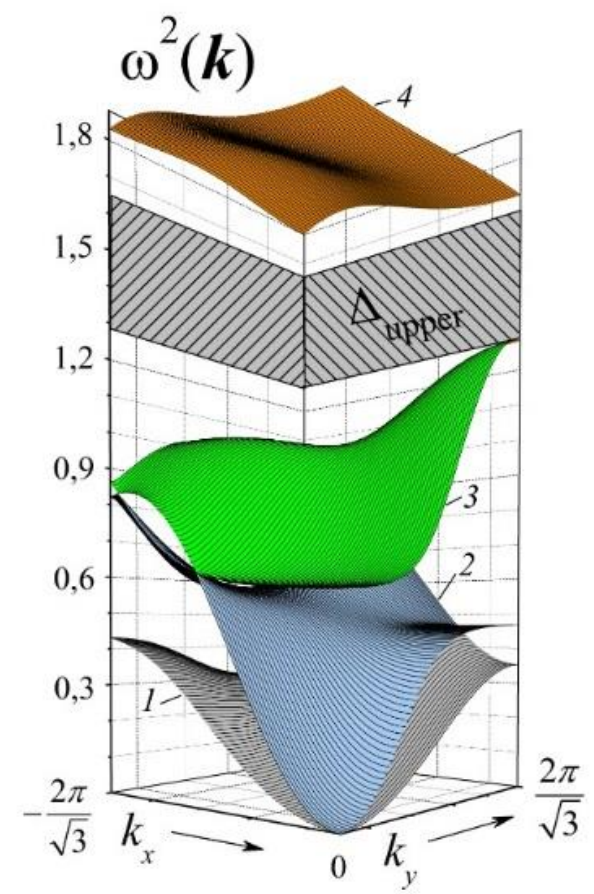

(a)

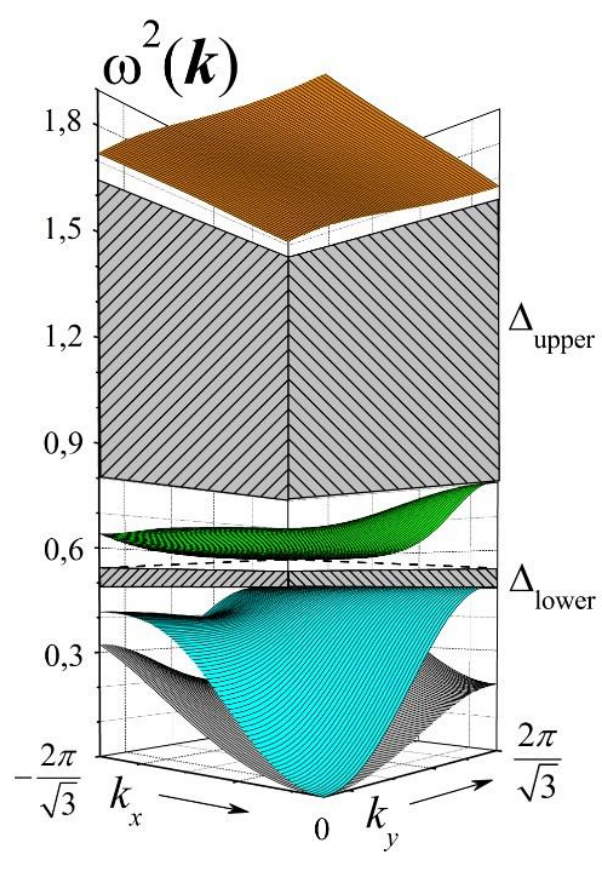

(b)

Fig. 14: Formation of gaps at the low value of parameter $\mu=\frac{m}{M}=0.1, \omega_{\text {int }, y}^{2}=0.55, \omega_{\text {int }, x}^{2}=1.65$. $a-\Omega_{0}^{2}=0.2, \Delta_{\text {upper }}=0.37 ; \mathrm{b}-\Omega_{0}^{2}=1 / 9, \Delta_{\text {upper }}=0.85, \Delta_{\text {lower }}=0.06$. The gaps are marked by grey dashed patterns.

Since $\Omega_{0}^{2}=\mu /\left(\frac{\chi_{i}}{\chi_{e}}\right)$, Eq. (22) can be re-written as:

$$
\frac{\chi_{i}}{\chi_{e}}>\frac{4 \mu}{1+\mu}
$$

Analogously, both the upper and lower gaps (between the frequency surfaces $2-3$ ) appear if the lower of the internal frequencies, $\omega_{i n t, y}^{2}=0.5(1+\mu)-($ See $8(b))-$ is greater than $\max \left\{\omega_{f, \text { upper }}^{2}(\boldsymbol{k})\right\}$ i.e. :

$$
\Omega_{0}^{2} \lesssim \frac{1+\mu}{12}, \frac{\chi_{i}}{\chi_{e}} \gtrsim \frac{12 \mu}{1+\mu} \cdot\left(\Omega_{0}^{2} \lesssim 0.09 \text { if } \mu=0.1\right)
$$

The thresholds set by Eqs. (22)-(24) are in a good agreement with the exact results (See. Fig. 15) at $\mu \lesssim 0.3$. When the parameter $\Omega_{0}^{2}$ decreases for a fixed value of $\mu\left(\frac{\chi_{i}}{\chi_{e}}\right.$ increases $)$, one can see that the formation of the upper gap precedes that of the lower gap and it first appears followed by the formation of the lower gap. Such an order of formation is comprehensible and could be expounded in accordance with the physical mechanisms of gaps formation presented above. 


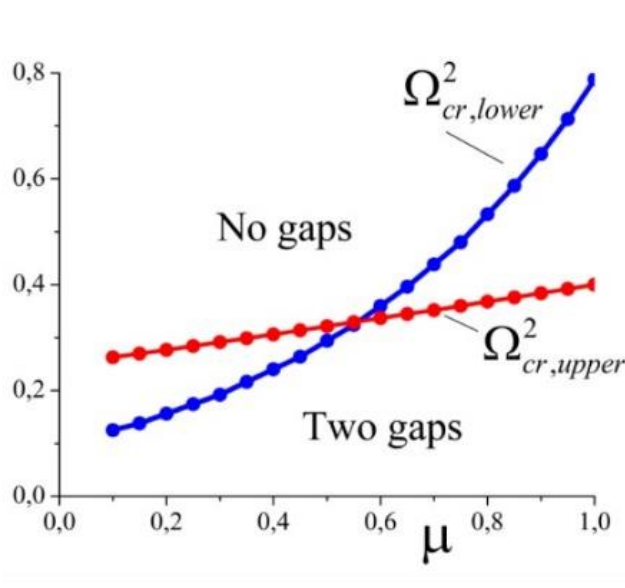

(a)

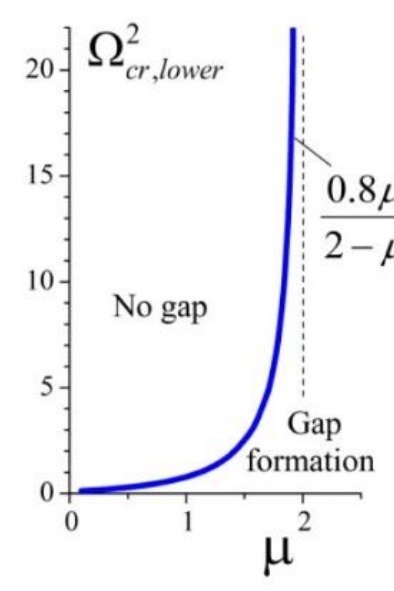

(b)

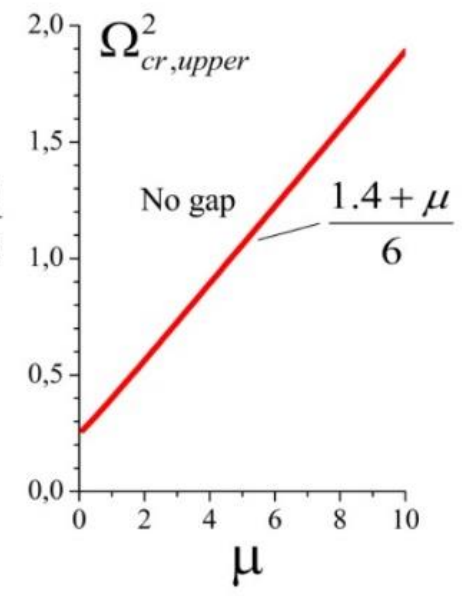

(c)

Fig. 15: The critical values of the parameter $\Omega_{0}^{2}$ as function of the mass ratio $\mu$. For the upper (lower) gap to be formed the relation $\Omega_{0}^{2}<\Omega_{c r \text {,upper }}^{2}\left(\Omega_{0}^{2}<\Omega_{c r, \text { lower }}^{2}\right)$ must hold. The region $0.1 \leq \mu \leq 1$ is presented separately in high resolution (Fig. 15a). The dependence $\Omega_{c r \text {,upper }}^{2}(\mu)$ is approximated by the linear function $\Omega_{c r, \text { upper }}^{2}=(1.4+\mu) / 6$ (Fig. 15c) with high accuracy as well as $\Omega_{c r, l o w e r}^{2}=$ $0.8 \mu /(2-\mu)$ at $\mu>0.8$ (Fig. 15b).

The order of appearance of gaps is only correct for low values of mass ratio (small internal mass) i.e. $m \ll M$. If $m \sim O(M)(\mu \gtrsim 0.6$, See Fig. 14) a sudden shift in the effect occurs. Then at first the lower gap forms (Compare Fig. 16 with Fig. 14).

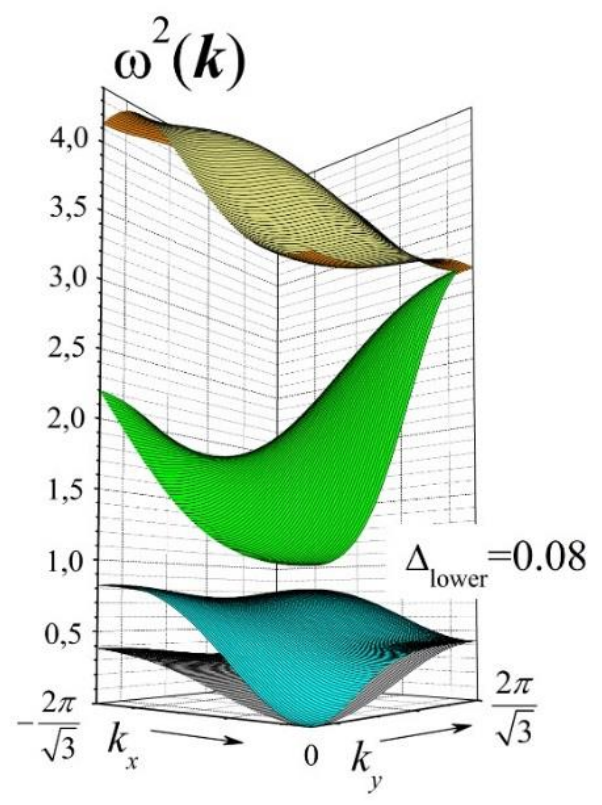

Fig. 16: The reverse order of gap formation when the parameter $\Omega_{0}^{2}$ is decreasing. $\mu=0.8, \Omega_{0}^{2}=0.45$. The upper gap will be formed at $\Omega_{0}^{2}<0.37$.

Our estimation of Eq. (22) $\Omega_{c r, \text { upper }}^{2}=(1+\mu) / 4-$ corresponds, at least qualitatively and approximately, to the exact result:

$$
\Omega_{\text {cr,upper }}^{2}(\mu)=(1.4+\mu) / 6
$$

But the threshold

$$
\Omega_{\text {cr,lower }}^{2}(\mu)=0.8 \mu /(2-\mu) .
$$

is not a linear function of mass ratio as Eq. (24) (i.e. $\Omega_{c r, \text { lower }}^{2}(\mu) \sim(1+\mu) / 12$ ) and demonstrates unusual behaviour (Fig. 15b). For $\mu>2$ the lower gap formally arises at any finite $\Omega_{0}^{2}$. 
More obviously, the properties of the acoustic system can be represented by the dependencies of the relative critical stiffness on the relative mass: $\left(\frac{\chi_{i}}{\chi_{e}}\right)_{c r}=\frac{\mu}{\Omega_{c r}^{2}(\mu)}$ - See. Fig. 17 (for the formation of gaps the inequality $\frac{\chi_{i}}{\chi_{e}}>\left(\frac{\chi_{i}}{\chi_{e}}\right)_{c r}$ must hold). One can see that at the region $\mu<2$ the lower gap can't appear if the relative internal stiffness is less than the critical value $\left(\frac{\chi_{i}}{\chi_{e}}\right)_{c r, l o w e r}$ derived.

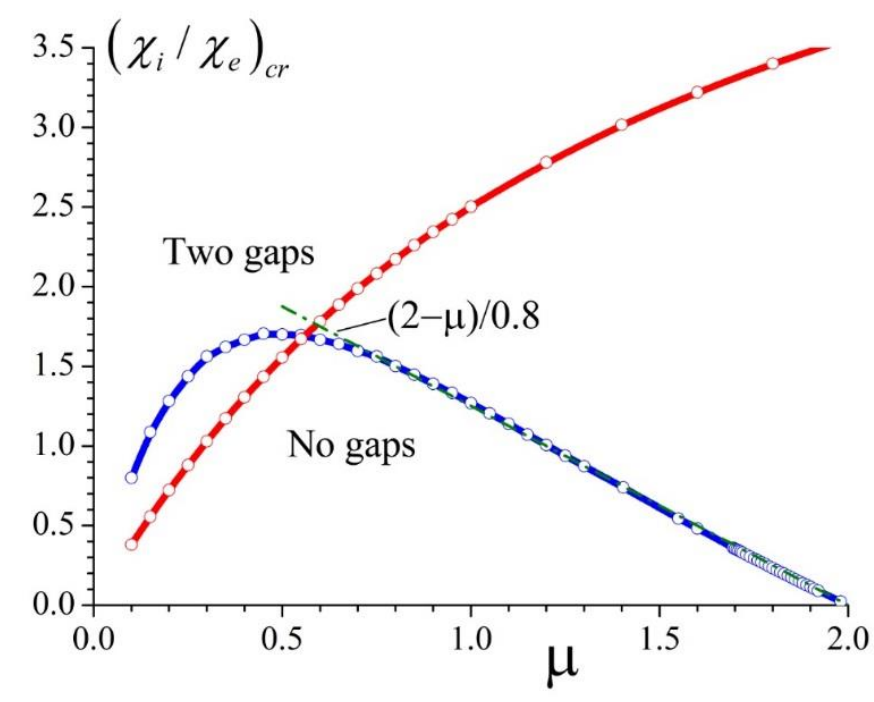

Fig. 17: The critical values of the parameter $\left(\chi_{i} / \chi_{e}\right)_{c r}$ as a function of the relative mass $\mu$. The blue curve is $\left(\chi_{i} / \chi_{e}\right)_{c r}$,lower , and the red $-\left(\chi_{i} / \chi_{e}\right)_{\text {cr,upper }}$. Gaps arise if the relative stiffness $\chi_{i} / \chi_{e}$ is higher than the corresponding critical value $\left(\chi_{i} / \chi_{e}\right)_{c r}$.

In accordance with the approximating function for $\Omega_{c r \text {, upper }}^{2}$ (See Eq. (25a))

$$
\left(\chi_{i} / \chi_{e}\right)_{\text {cr,upper }}=6 \mu /(1.4+\mu) \text {. }
$$

As a consequence of Eq. (26b) the value $\left(\frac{\chi_{i}}{\chi_{e}}\right)_{\text {cr,lower }}$ is a linear function of $\mu$ for $\mu \gtrsim 0.8$ :

$$
\left(\chi_{i} / \chi_{e}\right)_{\text {cr,lower }} \approx 1.25(2-\mu)
$$

At $\mu>\mu^{*}=2$, there aren't any limitations on $\chi_{i} / \chi_{e}$ for the formation of the lower gap. The threshold, $\mu^{*}$, has a clear explanation. Below, the value of $\mu^{*}$ for an arbitrary angle $\varphi$ between two internal springs is calculated - See Fig. 2a.

The dimensionless internal eigenfrequencies according to Eqs. (8a) and (8b) are equal to $\lambda_{x}^{2}=$ $2(1+\mu) \cos ^{2} \frac{\varphi}{2}, \lambda_{y}^{2}=2(1+\mu) \sin ^{2} \frac{\varphi}{2}$. Increasing the relative mass, $\mu$, is followed by increasing the dimensionless frequencies $\lambda_{x}^{2}$ and $\lambda_{y}^{2}$ at the fixed $\lambda_{x}^{2}\left(M^{*}=-\infty\right)=2 \cos ^{2} \frac{\varphi}{2}$. Thus, a possibility may be realized when:

$$
\lambda_{y}^{2}>\lambda_{x}^{2}\left(M^{*}=-\infty\right), \text { or } \mu>\mu^{*}=\cos ^{2} \frac{\varphi}{2} / \sin ^{2} \frac{\varphi}{2}-1
$$


In this case the lower of the two optical surfaces (surface 3) (See Fig. 12) lies above the $\max \left(\omega_{(2)}^{2}(\boldsymbol{k})\right)=\lambda_{x}^{2}\left(M^{*}=-\infty\right)$ (See Eq. (19)), thus Eq. (20) doesn't have a solution (the right hand side of Eq. (20) becomes negative at $\mu>\mu^{*}$ whereas the left hand side remains always positive). Such a situation guarantees that a gap appears above the upper acoustic frequency-surface (surface 2) and below the lower optic surface. The width of the gap, $\Delta_{\text {lower }}$, may be estimated as $\lambda_{y}^{2}-\lambda_{x}^{2}\left(M^{*}=\right.$ $-\infty)$ - (See Fig. 12). Then:

$$
\Delta_{\text {lower }}=2\left(\mu \times \sin ^{2} \frac{\varphi}{2}-\cos \varphi\right) .
$$

For $\varphi=\pi / 3$

$$
\mu^{*}=2, \Delta_{\text {lower }}=\frac{\mu}{2}-1,
$$

which is in agreement with the results presented in Fig. 18.

The results of Figs.17 and 18 show that there is a wide region on the plane $\left(\mu ; \chi_{i} / \chi_{e}\right)$ at which only the lower gap, $\Delta_{\text {lower }}$, can be formed with a rather large width. The width $\Delta_{\text {lower }}$ linearly increases when the relative mass, $\mu$, increases (Fig. 18a). The formation of only the upper gap is possible at a narrow zone (between red and blue curves in Fig. 17, $\mu \lesssim 0.6$ ). But this gap, $\Delta_{\text {upper }}$, don't exceed $\sim 0.78$ at the region.

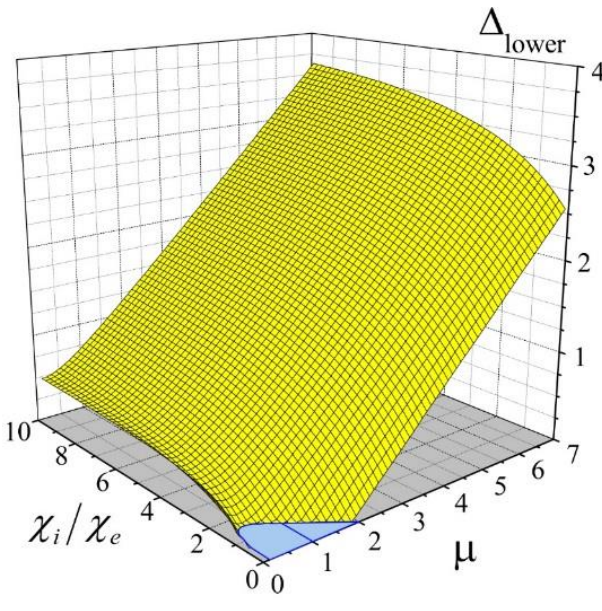

(a)

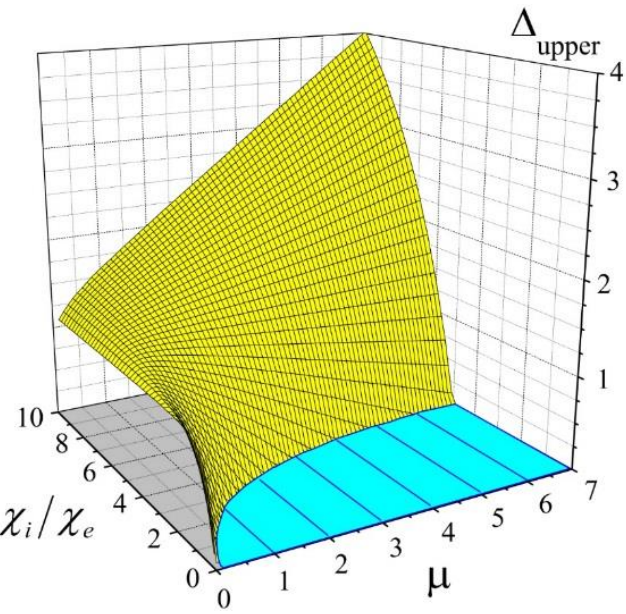

(b)

Fig. 18: The dependencies of $\Delta_{\text {lower }}\left(\mu ; \chi_{i} / \chi_{e}\right)$ and $\Delta_{\text {upper }}\left(\mu ; \chi_{i} / \chi_{e}\right)$ on the relative mass and dimensionless stiffness for $\mu \geq 0.1$, and $\chi_{i} / \chi_{e} \geq 0.1$. The regions where the gaps don't appear are marked by cyan.

As the results presented in Figs.18a and 18b depict, the single wide upper gap, $\Delta_{\text {upper }}>1$, can appear only together with a wide lower gap. Such an interconnection is demonstrated in Fig. 19: when $\Omega_{0}^{2}$ decreases $\left(\left(\chi_{i} / \chi_{e}\right)\right.$ increases $)$, significant widening of the upper gap doesn't affect the existing lower gap, which is mainly a function of $\mu$ (See Eq. (29)). But an interesting detail is to be noticed: the widening occurs because of "repulsion" between the upper (yellow) and the lower (green) frequency surfaces - the second one is localized in a more narrow frequency range in case Fig. 19b than in case of Fig. 19a. 


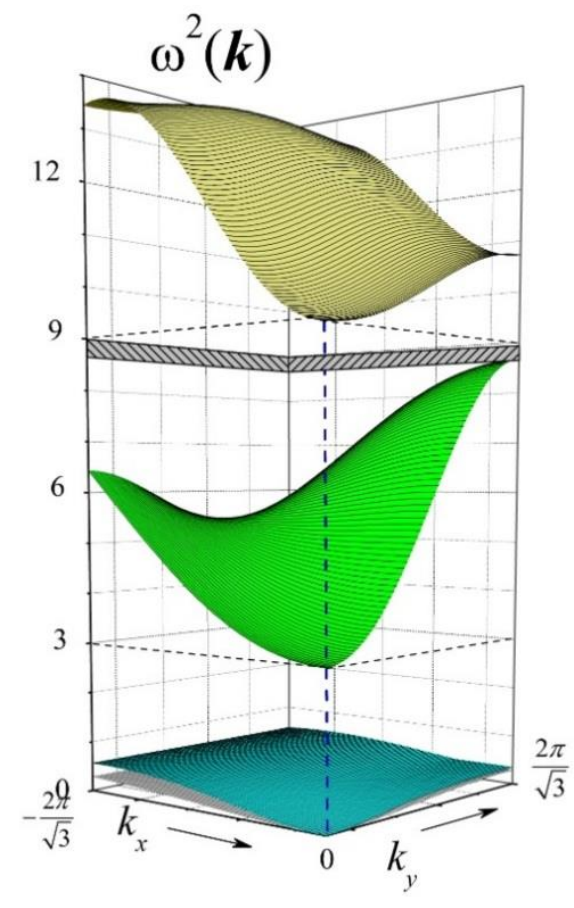

(a)

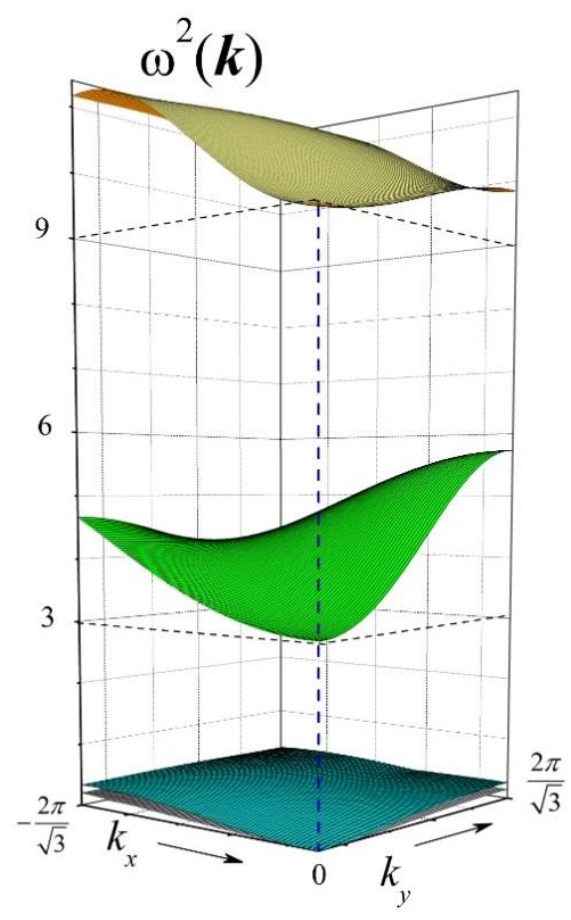

(b)

Fig. 19: Dispersion surface for $\mu=5\left(\lambda_{y}^{2}=3, \lambda_{x}^{2}=9\right) . a-\Omega_{0}^{2}=1\left(\Omega_{c r, u p p e r}^{2}=1.06 ;\left(\chi_{i}\right)\right.$ $\left.\left.\chi_{e}\right)_{\text {cr,lower }} \approx 4.7\right), \Delta_{\text {upper }}=0.35, \Delta_{\text {lower }}=2.44$ - the narrow upper gap is marked by gray pattern; $\mathrm{b}-\Omega_{0}^{2}=0.5\left(\chi_{i} / \chi_{e}=10\right), \Delta_{\text {upper }}=3.26, \Delta_{\text {lower }}=2.65$

So, on can taylor the width of the gaps, $\Delta_{\text {lower,upper }}$, through alteration of the governing parameters viz. $\mu, \beta=\chi_{i} / \chi_{e}$ (or alternatively $\Omega_{0}^{2}$ ) by using the results that are presented in Figs.15, 17 and 18.

There would be no reason to change the orientation of the internal springs at a fixed internal angle $\varphi$ between them since the main results practically don't change.

It would be instead more effective to change the angle $\varphi$ itself as shown by the Eq.'s (27)(28) to control the gap size $\Delta_{\text {lower }}$ (the ratio $\cos ^{2} \frac{\varphi}{2} / \sin ^{2} \frac{\varphi}{2} \equiv \lambda_{x}^{2} / \lambda_{y}^{2} \quad$ could be the meassure of anisotrophy of internal vibrations). But it is easy to ascertain that at $\varphi=60^{\circ}$ the magnitudes of $\Delta_{\text {lower }}$ and $\Delta_{\text {upper }}$ are the most balanced on average as they are of the same order of magnitude.

In the following the case of isotropy of the internal phononic structure leading to the global isotropy of the metamaterial is briefly studied.

\subsection{Homogeneous isotropic phononic metamaterial}

If $\varphi=90^{0}$, then $\lambda_{y}^{2}=\lambda_{x}^{2}=\lambda^{2}$, and internal vibrations become isotropic: vibrations oriented in any direction have the same frequency $\lambda^{2}=\frac{\chi_{i}}{m}(1+\mu)$ (or in the units of $\frac{\chi_{i}}{m}$ the dimensionless value $\lambda^{2}=1+\mu$ ).

As above, the maximum acoustic frequency, $\max \left(\omega_{(2)}^{2}\right)$, on surface 2 (Fig. 12) can't exceed the threshold: 


$$
\max \left(\omega_{(2)}^{2}\right)<\lambda^{2}\left(M^{*}=-\infty\right)=2 \cos ^{2} \frac{\varphi}{2}=1,
$$

(See Eqs. (19) for the derivation) and the critical mass parameter $\mu^{*}=0$ (see Eq. (27)). The physical meaning of such a low value of the relative critical mass, $\mu^{*}$, lies in the intensive "energy-level repulsion" which occurs between the isotropic acoustic and the twice-degenerate isotropic optical vibrations over the entire Brillion zone, which renders the appearance of contact points such as points $a, b, c$, and $d$ observed previously (See Fig. 9b, 10 and11) impossible.

Thus, the unique gap exists for any value of the parameters identifying the system under consideration:

$$
\Delta \approx \lambda^{2}-\lambda^{2}\left(M^{*}=-\infty\right)=\mu>0 .
$$

In fact, the gap $\Delta$ is slightly greater than $\mu$ because $\max \left(\omega_{(2)}^{2}\right) \approx \lambda^{2}\left(M^{*}=-\infty\right)$ only when $\Omega_{0}^{2}=$ $\mu /\left(\chi_{i} / \chi_{e}\right) \gg 1$. For a generic case when $\max \left(\omega_{(2)}^{2}\right)<\lambda^{2}\left(M^{*}=-\infty\right)$, the estimation (30) is in excellent agreement with numerical experiments (See Fig. 20).

Examples of frequency-surfaces are presented in Fig. 21. It is to be noticed an important relation. The internal vibrations tear the continuous spectrum of the two free acoustic vibrations $\left(\omega_{f, \text { lower }}^{2}(\boldsymbol{k}), \omega_{f, \text { upper }}^{2}(\boldsymbol{k})\right)$ into the pair of low-frequency acoustic oscillations, $\omega_{(1)}^{2}(\boldsymbol{k}), \omega_{(2)}^{2}(\boldsymbol{k})$, and the pair of high-frequency "optic" oscillations $\omega_{(3)}^{2}(\boldsymbol{k}), \omega_{(4)}^{2}(\boldsymbol{k})$. The results of our numerical experiments show that for these hybrid oscillations some conservation law is satisfied with high accuracy.

$$
\begin{aligned}
& \max \left[\omega_{(1)}^{2}(\boldsymbol{k})\right]+\left(\max \left[\omega_{(3)}^{2}(\boldsymbol{k})\right]-\lambda^{2}\right)=\max \left[\omega_{f, \text { lower }}^{2}(\boldsymbol{k})\right]=4.5 \Omega_{0}^{2}, \\
& \max \left[\omega_{(2)}^{2}(\boldsymbol{k})\right]+\left(\max \left[\omega_{(4)}^{2}(\boldsymbol{k})\right]-\lambda^{2}\right)=\max \left[\omega_{f, \text { upper }}^{2}(\boldsymbol{k})\right]=6 \Omega_{0}^{2}
\end{aligned}
$$

Where the left hand side depicts the conserved value and the right hand side in both are derived based on Eq. (12).

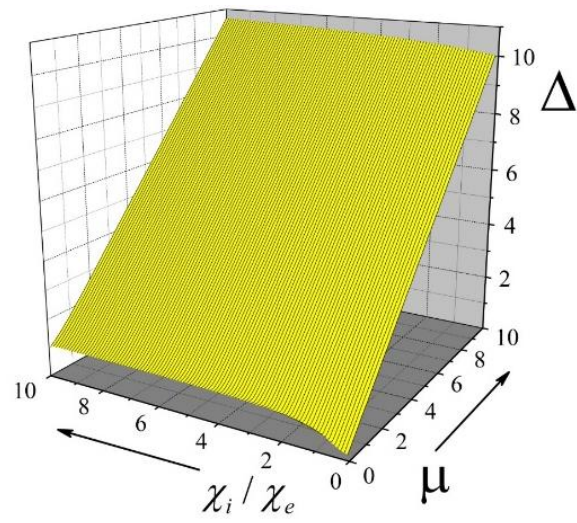

(a)

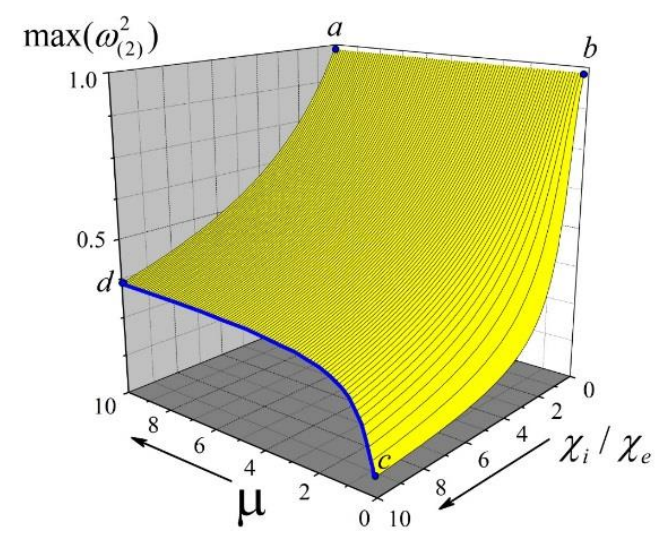

(b)

Fig. 20: The width of the gap $\Delta \approx \mu$ in accordance with (30) (especially for low values of $\chi_{i} / \chi_{e}$ ); For $\chi_{i} / \chi_{e} \gtrsim 1$ the frequency $\max \left(\omega_{(2)}^{2}\right)<\omega_{\text {int }}^{2}\left(M^{*}=-\infty\right)=1$, and for a fixed $\mu$ the gap $\Delta$ mainly increases due to the decrease in the value of $\max \left(\omega_{(2)}^{2}\right)$. The results shown are calculated for $\mu \geq$ $0.1, \chi_{i} / \chi_{e} \geq 0.1$. 


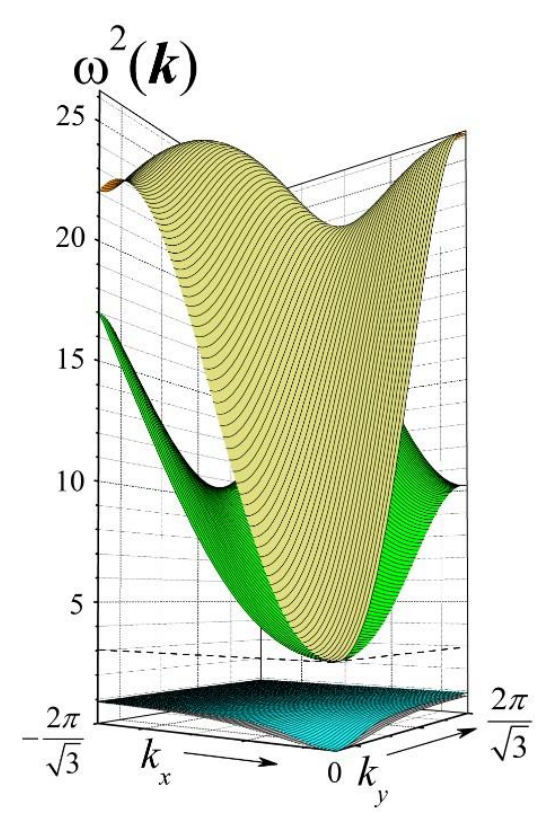

(a)

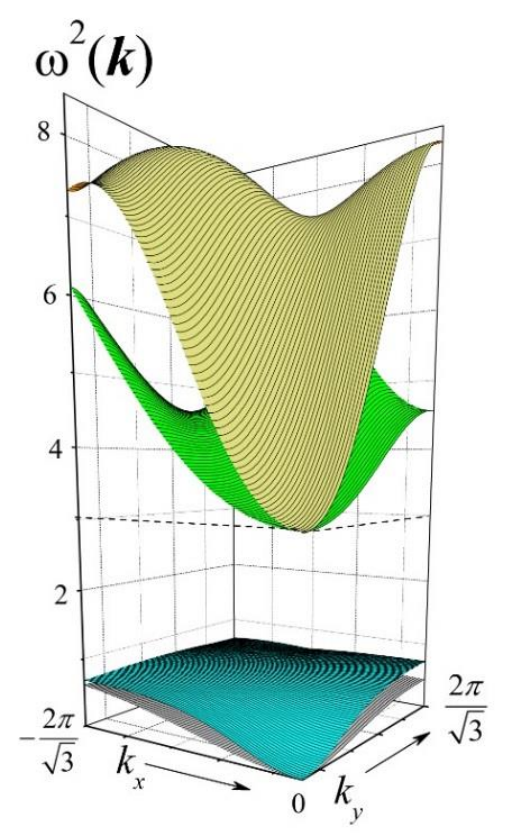

(b)

Fig. 21: Frequency surfaces for the isotropic case when (a) $\mu=2, \frac{\chi_{i}}{\chi_{e}}=0.5 ; \Omega_{0}^{2}=4$ and $\max \left(\omega_{2}^{2}\right) \approx \omega_{i n t, x}^{2}\left(M^{*}=-\infty\right)=1, \Delta=2.08$. (b) $\mu=2, \frac{\chi_{i}}{\chi_{e}}=2 ; \Omega_{0}^{2}=1, \max \left(\omega_{2}^{2}\right) \approx 0.725<$ $1, \Delta=2.275$.

In Eq. (31) it has been taken into account that $\omega_{(3)}^{2}(k=0)=\omega_{(4)}^{2}(k=0)=\lambda^{2}$. Eq. (31) could thus be interpreted as signifying the fact that the band of frequencies for each of the initial frequencysurfaces of supposed "free" external vibrations is torn (by the internal vibrations) into two single frequency-surfaces $\quad\left(\omega_{f, \text { lower }}^{2}(\boldsymbol{k}) \rightarrow \omega_{(1)}^{2}(\boldsymbol{k}) \oplus \omega_{(3)}^{2}(\boldsymbol{k})\right.$ and $\left.\quad \omega_{f, \text { upper }}^{2}(\boldsymbol{k}) \rightarrow \omega_{(2)}^{2}(\boldsymbol{k}) \oplus \omega_{(4)}^{2}(\boldsymbol{k})\right)$ with the same total width for the band of frequencies.

Furthermore, taking into account that $\max \left[\omega_{(1,2)}^{2}(\boldsymbol{k})\right]<1$ and using Eq. (31), one can estimate the band of frequencies of the "optical" vibrations, $\delta\left(\omega^{2}\right)$, as follows :

$$
\delta_{(3)}\left(\omega^{2}\right) \approx 4.49 \Omega_{0}^{2}-1 ; \delta_{(4)}\left(\omega^{2}\right) \approx 6 \Omega_{0}^{2}-1,
$$

Which is in good agreement with the data in Fig. 21.

\subsection{Asymptotic analyses $\left(\chi_{i} \rightarrow \infty, m \rightarrow \infty\right.$.)}

So far, the normalisation of frequencies has been done in all cases with respect to the unit of frequencies-squared i.e. $\frac{\chi_{i}}{m}$. (The eigenvalue of the free internal vibration if only one internal spring were involved). Now, let's suppose that the values of $M$ and $\chi_{e}$ are fixed as parameters $m$ and $\chi_{i}$ change. In this case it would be more convenient to use the value $\frac{\chi_{e}}{M}$ as the unit of frequenciessquared. The relation between the "old" $\left(\omega^{2}\right)$, and "new" $\left(\bar{\omega}^{2}\right)$ intrinsic frequencies of the system is obviously as follows: 


$$
\bar{\omega}^{2}=\omega^{2} \times\left(\chi_{i} / m\right) /\left(\chi_{e} / M\right)=\omega^{2} / \Omega_{0}^{2}, \bar{\Delta}=\Delta / \Omega_{0}^{2} .
$$

The results of Fig. 20 are then transformed to the results of Fig. 22 using Eq. (33)

Let's analyse the data in the light of new parameters defined. The exact value of the gap is $\Delta=1+\mu-\max \left[\omega_{(3)}^{2}(\boldsymbol{k})\right]$, therefore

$$
\bar{\Delta}=\left(1+\left(1-\max \left[\omega_{(2)}^{2}(\boldsymbol{k})\right]\right) / \mu\right) \times\left(\chi_{i} / \chi_{e}\right) .
$$

Formally, for $\mu \gg 1$, the inequality $\left(1-\max \left[\omega_{(3)}^{2}(\boldsymbol{k})\right]\right) / \mu \ll 1$ holds , and

$$
\bar{\Delta} \approx\left(\chi_{i} / \chi_{e}\right) .
$$

In fact, the gap $\bar{\Delta}$ can be estimated with an error which does not exceed $10 \%$ as follows:

$$
\bar{\Delta} \approx\left(1+\frac{1}{\mu}\right)\left(\frac{\chi_{i}}{\chi_{e}}\right) ; \quad \mu \geq 5, \chi_{i} / \chi_{e} \geq 5 .
$$

If $\mu=0.1$, then $\max \left[\omega_{(2)}^{2}(\boldsymbol{k})\right]$ (the path $b-c$ in Fig. 20b) can be approximated with a rather high accuracy as follows:

$$
\max \left[\omega_{(2)}^{2}(\boldsymbol{k})\right]=0.55 /\left(\chi_{i} / \chi_{e}\right), \quad \chi_{i} / \chi_{e} \geq 1
$$

Accordingly, $\max \left[\bar{\omega}_{(2)}^{2}(\boldsymbol{k})\right] \approx 5.5$ for $\chi_{i} / \chi_{e} \geq 1$ - See the line b-c in Fig. 22b.

Then, using Eq. (34),

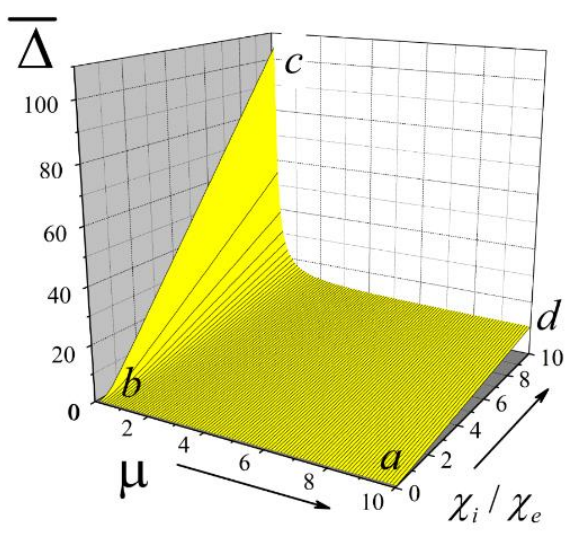

(a)

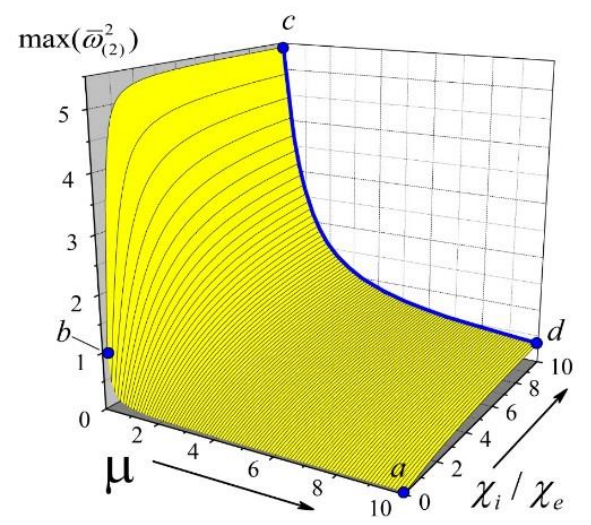

(b)

Fig. 22: The width of gap and $\max \left(\omega_{2}^{2}\right)$ presented in unit of $\chi_{e} / M$ as functions of $\mu$ and $\chi_{i} / \chi_{e}$ at fixed values $\chi_{e}$ and $M$. The point $b$ calculated at $\mu=0.1, \frac{\chi_{i}}{\chi_{e}}=0.1$.

$$
\bar{\Delta}(\mu=0.1)=\left(1+\frac{1}{\mu}\right)\left(\frac{\chi_{i}}{\chi_{e}}\right)-\frac{0.55}{\mu}=11\left(\frac{\chi_{i}}{\chi_{e}}\right)-5.5 ; \quad \chi_{i} / \chi_{e} \geq 1 .
$$

The linear dependence expressed in Eq. (38) can be seen in Fig. $22 a$ i.e. the line $b$-c. 
Finally, a word on the physical meaning of the blue line $c-d$ in Fig. 22b (or analogously in Fig. 20b) is in order. The surface $\omega_{(2)}^{2}(\boldsymbol{k})$ presents the upper branch of acoustic vibrations. For a suitably high value of the stiffness of internal springs, oscillations may be excited in which every node can be regarded as a single node of the total mass $M_{t o t} \sim(m+M)=M(1+\mu)$. Thus, at high values of $\frac{\chi_{i}}{\chi_{e}} \gg$ 1 the frequency of acoustic oscillations must be of the order of $1 / M_{\text {tot }}$. For the fixed $M$, as stated above,

$$
\max \left[\bar{\omega}_{(2)}^{2}(\boldsymbol{k})\right] \sim 1 /(1+\mu) ; \quad\left(\frac{\chi_{i}}{\chi_{e}}\right) \gg 1 .
$$

The dependency (39) is in good agreement with the result shown in Fig. 22b - the blue line $c$ $d$ when $\left(\frac{\chi_{i}}{\chi_{e}}\right)=10$.

\section{Conclusions}

The present study focuses on different aspects of band structure and dispersion surfaces in metamaterials of a particular topology $\left(\mathrm{K}_{3}\right)$. On the basis of mechanical equations for single nodes of the $\mathrm{K}_{3}$-acoustic system the simple analytical form of the dispersion matrix $\boldsymbol{D}\left(\omega^{2}, \boldsymbol{k}\right)$ has been constructed, which provides a convenient means for some qualitative and quantitative estimations to be made. A significant result is the statement about existence of the absolutely different mechanisms of gaps formation between acoustic, $\omega_{(1),(2)}^{2}(\boldsymbol{k})$, and optical, $\omega_{(3),(4)}^{2}(\boldsymbol{k})$, surface frequencies. Sometimes this formation is the bright display of quantum effects like strong coupling, energy splitting, and level crossings in classical mechanical systems (See for example [43]).

Formally, the parameter $\mu$ in the dynamic matrix i.e. $\boldsymbol{D}(\omega, \boldsymbol{k})$-matrix is the key parameter as it determines the strength of interaction between the free internal vibrations (FIV) and free external vibrations (FEV). (When $\mu$ alters, the reference frequency $\omega_{0}^{2}=$ is supposed to be fixed). Qualitatively, for invariable total mass, $M_{t o t}=M+m$, and given energy of the FIV the displacements of a node's shell, $\boldsymbol{u}_{\text {int,e }}$, are proportional to the ratio $m / M \equiv \mu$. So, the effects of interference between $\boldsymbol{u}_{\text {int,e }}$ and $\boldsymbol{u}_{f, e}$, which are responsible for the formation of gaps, intensify as $\mu$ increases. Only at single points where $\boldsymbol{u}_{i n t, e}(\omega, \boldsymbol{k}) \perp \boldsymbol{u}_{f, e}(\omega, \boldsymbol{k})$ the level repulsion doesn't push the surface-frequencies of different types aside and these surfaces touch each other.

There are two extreme cases of gap formation between the upper acoustic mode $\omega_{(2)}^{2}(\boldsymbol{k})$, and the lower optical mode $\omega_{(3)}^{2}(\boldsymbol{k})\left(\varphi<90^{\circ}\right)$. These are expounded as follows:

A. The case of small relative mass $\mu \ll 1(m \ll M)$. Then in order to avoid overlapping of the bands of frequencies $\delta \omega_{(2)}^{2}(\boldsymbol{k})$ and $\delta \omega_{(3)}^{2}(\boldsymbol{k})$ the parameter $\Omega_{0}^{2}\left(\sim \chi_{e}\right)$ must be low enough so that the upper of the free external vibration frequency, $\max \left(\omega_{f, \text { upper }}^{2}\right)$, be of the order of, or less than the internal vibrations frequency, $\lambda_{y}^{2}$. Gap formation under these conditions corresponds to the minimal strength of coupling between the internal and external vibrations. The low coupling results in the narrow bands of frequencies $\delta \omega_{(3)}^{2}(\boldsymbol{k}), \delta \omega_{(4)}^{2}(\boldsymbol{k})$ as compared to that for the acoustic modes, $\delta \omega_{(1)}^{2}(\boldsymbol{k}), \delta \omega_{(2)}^{2}(\boldsymbol{k})$. The displacements on the optical frequency surfaces 3 and $4, \boldsymbol{u}_{e}^{(3)}(\omega, \boldsymbol{k}), \boldsymbol{u}_{e}^{(4)}(\omega, \boldsymbol{k})$, (where $\boldsymbol{u}_{e}^{(i)}(\omega, \boldsymbol{k}), i=1,2,3,4-$ are the eigenvectors of the matrix $\boldsymbol{D}(\omega, \boldsymbol{k}))$ are mainly oriented along $k_{y}$-and $k_{x}$-axis 
correspondingly. That is, the optical vibrations have anisotropic polarization relative to the wave vector.

B. The case of large relative mass $\mu>1(m>M)$. Large internal mass gives rise to enhanced coupling between the internal and external vibrations and the strength of coupling rises sharply. At $\mu>\mu^{*}$ the optical vibrations break the continuous spectrum of "acoustic phonons" creating the gap $\Delta_{\text {lower }} \approx \lambda_{y}^{2}-\lambda_{x}^{2}\left(M^{*}=-\infty\right)$ for any parameters $\Omega_{0}^{2}$. However, the bigger this parameter, the wider is the bands of frequencies $\delta \omega_{(3)}^{2}(\boldsymbol{k}), \delta \omega_{(4)}^{2}(\boldsymbol{k})$ relative to the maximal acoustic frequency. (The region of low values of $\Omega_{0}^{2}$ (low $\chi_{e}$ ) is not so interesting because the formations of the two gaps $\omega_{(2)}^{2}(\boldsymbol{k}) \Leftrightarrow \omega_{(3)}^{2}(\boldsymbol{k})$ and $\omega_{(3)}^{2}(\boldsymbol{k}) \Leftrightarrow$ $\omega_{(4)}^{2}(\boldsymbol{k})$ occur with the scenario presented below as section A. ) By changing the parameter $\Omega_{0}^{2}$ we can significantly change the polarization properties of the optical oscillations, $\omega_{(3)}^{2}(\boldsymbol{k}), \omega_{(4)}^{2}(\boldsymbol{k})$ (See Fig. 23, for example).

At $\Omega_{0}^{2}=4$ only in the central region of the first Brillouin zone (the dark yellow circle) the polarization is extensively anisotropic (Fig. 23b) contrary to the case when $\Omega_{0}^{2}=1$ (Fig. 23a) with marked anisotropy over the entire Brillouin zone (the same effects can be observed for the displacements $\left.\boldsymbol{u}_{e}^{(4)}(\omega, \boldsymbol{k})\right)$. So, one can tune the governing filtering properties of the optical branches by alteration of defining parameters.

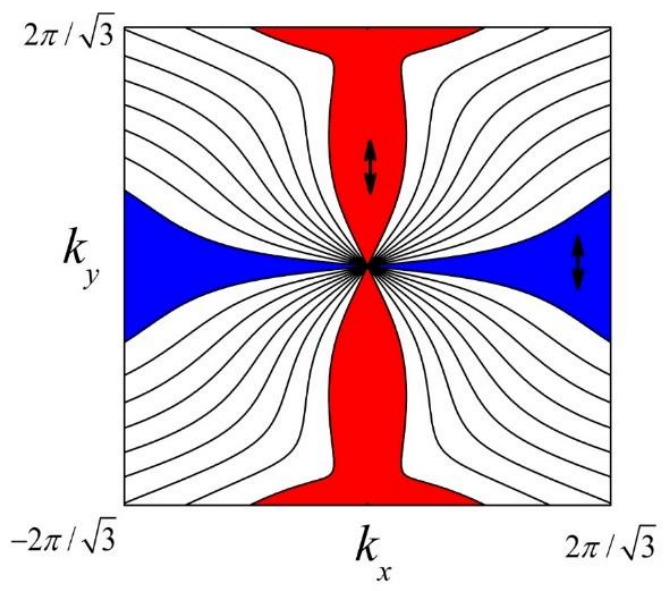

(a)

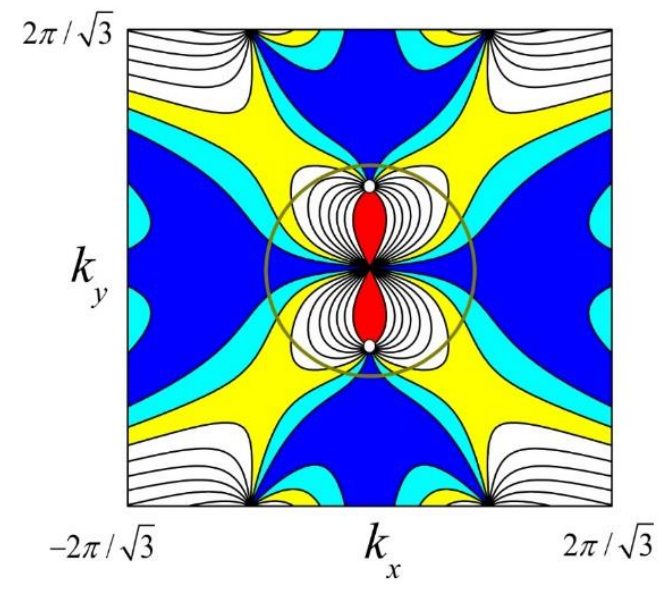

(b)

Fig. 23: Distributions of the parameter of polarization for the lower branch of optical vibrations in the first Brillouin zone: $\Phi(k)=\left(\boldsymbol{u}_{e}^{(3)}(\omega, \boldsymbol{k}) \cdot \boldsymbol{k}\right) /\left(|\boldsymbol{k}|\left|\boldsymbol{u}_{e}^{(3)}\right|\right) \equiv \cos \alpha, \alpha$ is the angle of polarization. $\varphi=60^{0}, \mu=3$. (a) $\Omega_{0}^{2}=1, \delta \omega_{(2)}^{2}(\boldsymbol{k}) \approx 0.74, \delta \omega_{(3)}^{2}(\boldsymbol{k}) \approx 5.6, \Delta_{\text {lower }} \approx 1.26$; in the red regions $-0.9 \leq \Phi(k) \leq 1$, in the blue regions $0 \leq \Phi(k) \leq 0.1$, black arrows show the typical displacements on the lower optical surface. (b) $\Omega_{0}^{2}=4, \delta \omega_{(2)}^{2}(\boldsymbol{k}) \approx 1.21, \delta \omega_{(3)}^{2}(\boldsymbol{k}) \approx 19$, $\Delta_{\text {lower }}=0.8$; regions of cyan $-0.1 \leq \Phi(k) \leq 0.2$, regions of yellow $0.2 \leq \Phi(k) \leq 0.3$, white hollow circles signify the singular points with undefined polarization. 
C. The case of isotropic internal vibrations is distinguished by its conspicuous ability to form the lower gap $\Delta_{\text {lower }}$ at any $\mu>0$. This phenomenon is based on the strongest coupling of the isotropic acoustic and the twice-degenerate optical vibration on intersections of $\boldsymbol{u}_{f, e}^{(0)}(\boldsymbol{k})$ and $\boldsymbol{u}_{i n t, e}^{(0)}\left(\boldsymbol{u}_{f, e}^{(0)}(\boldsymbol{k})\right.$ is the non-perturbed displacement of the external mass as $\mu \rightarrow 0, \beta \rightarrow$ 0 while $\omega_{0}^{2}=$ const ; and $\boldsymbol{u}_{\text {int }, e}^{(0)}-$ that for $\chi_{e}=0$ ).

So, in the present study the fundamental characteristics of gap formation for $\mathrm{K}_{3}$-phononic metamaterials have been discussed. The different dependencies of band structure and dispersion surfaces on model parameters were quantitatively established and expounded. The methods to control gap parameters and properties of the optical vibrations created over these gaps were investigated.

\section{Acknowledgments}

The authors wish to express their gratitude for the financial support provided by the British councilKiev under the Academic Partnership/Mobility Grant scheme grant No. UKR16EG/3/19.01.16.

\section{Bibliography}

1. Pendry, J.B., Negative Refraction Makes a Perfect Lens. Physical Review Letters, 2000. 85(18): p. 3966-3969.

2. Smith, D.R., J.B. Pendry, and M.C.K. Wiltshire, Metamaterials and negative refractive index. Science, 2004. 305(5685): p. 788-792.

3. Veselago, V., et al., Negative refractive index materials. Journal of Computational and Theoretical Nanoscience, 2006. 3(2): p. 189-218.

4. Veselago, V.G., Waves in metamaterials: their role in modern physics. Physics-Uspekhi, 2011. 54(11): p. 1161-1165.

5. Veselago, V.G., Energy, linear momentum, and mass transfer by an electromagnetic wave in a negative-refraction medium. Physics-Uspekhi, 2009. 52(6): p. 649-654.

6. Veselago, V.G., Some remarks regarding electrodynamics of materials with negative refraction. Applied Physics B-Lasers and Optics, 2005. 81(2-3): p. 403-407.

7. Veselago, V.G., Electrodynamics of materials with negative index of refraction. PhysicsUspekhi, 2003. 46(7): p. 764-768.

8. Veselago, V.G., Electrodynamics of media with simultaneously negative electric permittivity and magnetic permeability. Advances in Electromagnetics of Complex Media and Metamaterials, 2002. 89: p. 83-97.

9. Linden, S., et al., Magnetic response of metamaterials at 100 terahertz. Science, 2004. 306(5700): p. 1351-1353.

10. Vukusic, P. and J.R. Sambles, Photonic structures in biology. Nature, 2003. 424(6950): p. 852855.

11. Padilla, W.J., D.N. Basov, and D.R. Smith, Negative refractive index metamaterials. Materials Today, 2006. 9(7-8): p. 28-35.

12. Li, J. and C.T. Chan, Double-negative acoustic metamaterial. Physical Review E, 2004. 70(5): p. 055602.

13. Poulton, C.G., Eigenvalue problems for doubly periodic elastic structures and phononic band gaps. Proceedings - Royal Society. Biological sciences, 2000. 456(2002): p. 2543.

14. Kushwaha, M.S., et al., Acoustic Band-Structure of Periodic Elastic Composites. Physical Review Letters, 1993. 71(13): p. 2022-2025. 
15. Kushwaha, M.S., et al., Theory of Acoustic Band-Structure of Periodic Elastic Composites. Physical Review B, 1994. 49(4): p. 2313-2322.

16. Martinsson, P.G. and A.B. Movchan, Vibrations of lattice structures and phononic band gaps. Quarterly Journal of Mechanics and Applied Mathematics, 2003. 56: p. 45-64.

17. Liu, X.N., et al., Wave propagation characterization and design of two-dimensional elastic chiral metacomposite. Journal of Sound and Vibration, 2011. 330(11): p. 2536-2553.

18. Huang, G.L., Band gaps in a multiresonator acoustic metamaterial. Journal of vibration and acoustics, 2010. 132(3): p. 31003.

19. Huang, H. and C. Sun, Wave attenuation mechanism in an acoustic metamaterial with negative effective mass density. New journal of physics, 2009. 11(1): p. 013003.

20. Veselago, V.G., Formulating Fermat's principle for light traveling in negative refraction materials. Physics-Uspekhi, 2002. 45(10): p. 1097-1099.

21. Zalipaev, V.V., A.B. Movchan, and I.S. Jones, Two-parameter asymptotic approximations in the analysis of a thin solid fixed on a small part of its boundary. The Quarterly Journal of Mechanics and Applied Mathematics, 2007. 60(4): p. 457-471.

22. Martinsson, P.G., Vibrations of lattice structures and phononic band gaps. The Quarterly Journal of Mechanics and Applied Mathematics, 2003. 56(1): p. 45.

23. Wang, G., et al., Lumped-mass method for the study of band structure in two-dimensional phononic crystals. Physical Review B, 2004. 69(18).

24. Li, J. and C.T. Chan, Double-negative acoustic metamaterial. Physical Review E, 2004. 70(5).

25. Veselago, V.G., The electrodynamics of substances with simultaneously negative values of $\varepsilon$ and $\mu$. Physics-Uspekhi, 1968. 10(4): p. 509-514.

26. Liu, Z., Analytic model of phononic crystals with local resonances. Physical review. B, Condensed matter, 2005. 71(1): p. 014103.

27. A. Srikantha Phani, J.W., and N. A. Fleck, Wave propagation in two-dimensional periodic lattices. J. Acoust. Soc. Am., 2006. 119( 4): p. 1995-2005.

28. Yang, Y., A.S. Fallah, and L.A. Louca, Frequency analysis of a heterogeneous perforated panel using a super-element formulation. Journal of Sound and Vibration, 2009. 327(1-2): p. 26-40.

29. Toolabi, M., et al., Dynamic analysis of a viscoelastic orthotropic cracked body using the extended finite element method. Engineering Fracture Mechanics, 2013. 109: p. 17-32.

30. Fallah, A.S., et al., Wave propagation in two-dimensional anisotropic acoustic metamaterials of K4 topology. Wave Motion, 2015. 58: p. 101-116.

31. Kittel, C., Elementary Solid State Physics: A Short Course. 1962, New York: Wiley.

32. Brillouin, L.o., Wave propagation in periodic structures; electric filters and crystal lattices. $2 \mathrm{~d}$ ed. 1953, New York: Dover Publications. xii, 255 p.

33. Born, M. and K. Huang, Dynamical theory of crystal lattices. Oxford classic texts in the physical sciences. 1988, Oxford New York: Clarendon Press ; Oxford University Press. xii, 420 p.

34. Sutton, A.P., Electronic structure of materials. Oxford science publications. 1993, Oxford New York: Clarendon Press ; Oxford University Press. xv, 260 p.

35. Sigmund, O. and J.S. Jensen, Systematic design of phononic band-gap materials and structures by topology optimization. Philosophical Transactions of the Royal Society of London Series a-Mathematical Physical and Engineering Sciences, 2003. 361(1806): p. 10011019.

36. Matlack, K.H., et al., Composite 3D-printed metastructures for low-frequency and broadband vibration absorption. Proceedings of the National Academy of Sciences, 2016. 113(30): p. 8386-8390.

37. Maldovan, M., Sound and heat revolutions in phononics. Nature, 2013. 503(7475): p. 209217. 
38. Kaveh, A., Structural mechanics : graph and matrix methods. 2nd ed. Applied and engineering mathematics series. 1995, Taunton, Somerset, England New York: Research Studies Press ; Wiley. xv, 440 p.

39. Tutte, W.T., Graph theory. Encyclopedia of mathematics and its applications. 1984, Cambridge Cambridgeshire ; New York, NY, USA: Cambridge University Press. xxi, 333 p.

40. Wagner, K., Über eine Eigenschaft der ebenen Komplexe. Mathematische Annalen, 1937. 114(1): p. 570-590.

41. Landau, L.D. and E.M. Lifshits,'Quantum mechanics : non-relativistic theory. $3 \mathrm{~d}$ ed. Their Course of theoretical physics. 1977, Oxford ; New York: Pergamon Press. xiv, 673 p.

42. von Neumann J., W.E.P., Ueber merkwuerdige diskrete Eigenwerte. Z. Phys., , 1929. 30 p. 465-467.

43. Novotny, L., Strong coupling, energy splitting, and level crossings: A classical perspective. American Journal of Physics, 2010. 78(11): p. 1199-1202. 